CONFORMAL GEOMETRY AND DYNAMICS

An Electronic Journal of the American Mathematical Society

Volume 10, Pages 227-256 (September 26, 2006)

S $1088-4173(06) 00141-\mathrm{X}$

\title{
GHYS-LIKE MODELS FOR LAVAURS AND SIMPLE ENTIRE MAPS
}

\author{
ARNAUD CHÉRITAT
}

\begin{abstract}
We provide a new geometric construction of pre-models (à la Ghys) for Lavaurs maps, from which we deduce that their Siegel disk is a Jordan curve running through a critical point, which was not known before. The construction turns out to work also for a class of entire maps, very specific, nonetheless including cases where no pre-models were known.
\end{abstract}

\section{Contents}

1. Introduction

1.1. In short

1.2. Historical background

1.3. Overview of the article

2. The classical surgery

3. The geometric construction of the pre-model

3.1. For quadratic polynomials

3.2. For some entire maps

3.3. For some Lavaurs maps

4. From the pre-model to the model

5. Rigidity

5.1. Entire maps

5.2. Lavaurs maps

6. The straightening

6.1. Entire maps

6.2. Lavaurs maps

6.3. Illustrations

7. Uncountably many inequivalent entire maps

8. Getting more control for Lavaurs maps

9. Objects of the parabolic implosion

10. Conclusion

Acknowledgments

References

Received by the editors June 1, 2005.

2000 Mathematics Subject Classification. Primary 37F40, 37F50; Secondary 37F10.

(C)2006 American Mathematical Society Reverts to public domain 28 years from publication 


\section{INTRODUCTION}

1.1. In short. For quadratic polynomials with an indifferent fixed point with bounded type rotation number, much of what is known of their Siegel disk and of their Julia set comes from the study of a quasiconformal model. The model is built from a Blaschke fraction (that we call a pre-model), that is usually defined by a formula. Beyond the quadratic maps, there are some other families for which models are known, whose pre-models are often defined by their formula. We give here a new geometric construction of pre-model maps. This construction covers several cases for which no models were known to exist. More precisely, we are able to make the model work: first for a class of entire maps, very specific but nonetheless spanning uncountably many equivalence classes (thus with probably no hope for a formula), second in the case of the Lavaurs maps that arise in the parabolic implosion of quadratic polynomials. Only the most immediate corollaries of this model are derived: e.g. that the Siegel disk is a Jordan curve running through a critical point (which was unknown before, in the case of Lavaurs maps).

1.2. Historical background. The first examples of Siegel disks with Jordan curve boundary were given by Herman He1. They are provided by a quasiconformal surgery procedure due to Ghys Gh. The surgery was done on a degree 3 Blaschke fraction. This gave examples of polynomial, rational and entire maps with a Siegel disk whose boundary is a bounded Jordan curve, which does not contain any critical point.

Douady remarked in a Bourbaki seminar D1] in 1987 that one should be able to apply the same procedure to a model with bounded type rotation number and a critical point on the boundary of the Siegel disk, depending on a conjecture related to critical circle maps. Herman $\underline{\mathrm{He} 2}$, using inequalities by Świątek $\underline{\mathrm{Sw}}$, proved this conjecture 1 . This is the construction that we extend in this article.

The quasiconformal model not only leads to a better understanding of the Siegel disk of the concerned maps, but also of its Julia set (concerning its Hausdorff dimension and its local connectivity for instance): see for instance [P1], $\mathrm{mM}$ ], and work of Lyubich. However, these questions are beyond the scope of this article.

Remark. It is worth mentioning that these surgeries prove the linearizability of $P$ at the fixed point 0, without using Siegel's linearization theorem.

Working with other models enables one to transfer these results to other holomorphic maps. See for instance [YaZ and Ge, who work with an explicit model. For a general rational map, Shishikura has an approach to provide models by contracting Herman rings, which has so far remained unpublished.

Does any function with a bounded type indifferent fixed point have a model? We provide a positive answer for functions having some form of rigidity.

1.3. Overview of the article. In section 2, we briefly recall the classical surgery done in $\mathrm{He} 2$.

It can be viewed as follows:

$$
\text { pre-model } \longrightarrow \text { model } \longrightarrow \text { dynamics }
$$

${ }^{1}$ PZ] gave a spectacular improvement in terms of the set of rotation numbers covered by the surgery, but we will not deal with it here. 
which means the following: Starting from an analytic map (the pre-model) which restricts to a critical circle map 2 one first post-composes it with a rotation, so that the rotation number is of bounded type, and then constructs a quasiregular map (the model), replacing the dynamics inside the unit disk with a quasiconformal rotation, provided by Herman-Świątek and Ahlfors-Beurling. Then by defining an invariant Beltrami form and straightening it, the model gets conjugated to an analytic map, whose dynamics one studies using the model.

It shall be noted that the pre-model is often given a priori, by a formula. We take a different approach.

In section 3, we introduce our geometric construction. We first present it on the simple case of the quadratic polynomial, which inspired our construction. In increasing order of difficulty, we then explain the construction for some entire maps, and last for the Lavaurs maps.

Our construction starts from a holomorphic map and builds from it another holomorphic map preserving the unit circle:

$$
\text { dynamics } \longrightarrow \text { pre-model. }
$$

Then, the classical surgery creates from this new dynamics having the same combinatorial type. The new dynamics needs not be the map we started from. However, it is the same in a few cases where there is enough rigidity.

Rigidity lemmas are given in section 5, and used in section 6 to prove that we indeed get the same map. As an immediate corollary, in the families we will consider, every time the rotation number has bounded type we know that the Siegel disk is bounded by a Jordan curve containing a critical point.

In section 7 we prove that the families of entire maps for which our construction works spans uncountably many topological equivalence classes. This shows that our approach covers many cases where there is no hope for a pre-model given by a simple formula.

In section 8 we prove that for a given bound on the entries of the continued fraction of the bounded type rotation number, there is some definite modulus between the Siegel disk and the boundary of the domain of definition of the Lavaurs map.

The notations and definitions of the theory of Lavaurs maps are recalled in section 9 ,

\section{THE ClASSiCAL SURGERY}

We will call $\mathbb{S}^{1}$ the unit circle in the complex plane $\mathbb{C}$, and $\mathbb{S}^{2}$ the Riemann sphere. Let $B$ be the rational map given by the formula

$$
B(z)=z^{2} \frac{z-3}{1-3 z} \text {. }
$$

This is a Blaschke fraction: $B\left(\mathbb{S}^{1}\right)=\mathbb{S}^{1}$. The restriction of $B$ to $\mathbb{S}^{1}$ is an orientation preserving homeomorphism. It is not a diffeomorphism, since the derivative vanishes at $z=1$. The local degree of this critical point is 3 . Let us call $B$ the pre-model.

Let $R_{\tau}$ be the rotation of center 0 and rotation number $\tau: R_{\tau}(z)=e^{i 2 \pi \tau} z$. We introduce the family $B_{\tau}=R_{\tau} \circ B$. According to the theory of rotation numbers

\footnotetext{
${ }^{2}$ bijective, orientation preserving, but with the derivative vanishing at least once
} 
(see for instance $[\mathrm{dMvS}$ ), for any irrational $\theta \in \mathbb{R} / \mathbb{Z}$, there is one and only one value of $\tau \in \mathbb{R} / \mathbb{Z}$ such that $B_{\tau}$ has rotation number $\theta$ on $\mathbb{S}^{1}$. According to the HermanŚwiatek theorem 3 , the restriction of $B_{\tau}$ to $\mathbb{S}^{1}$ is quasisymmetrically conjugated to $R_{\theta}$ if and only if $\theta$ has bounded type. If this is the case, then according to the Ahlfors-Beurling theorem 4 , the conjugacy extends to a quasiconformal selfhomeomorphism $\phi$ of the unit disk. The model $\widetilde{B}$ is defined by

$$
\begin{aligned}
\widetilde{B}(z)= & B(z) & & \text { if } z \in \mathbb{S}^{2} \backslash \mathbb{D}, \\
& \phi^{-1} \circ R_{\theta} \circ \phi(z) & & \text { if } z \in \mathbb{D} .
\end{aligned}
$$

This map is quasiregular. It is a ramified covering of degree 2, with critical points $\infty$ and 1 . Let $\mu_{0}$ be the pull-back by $\phi$ of the null Beltrami form on $\mathbb{D}$, and $\mu$ be the following $\widetilde{B}$-invariant Beltrami form: For $z \in \mathbb{S}^{2}$, either the orbit of $z$ under $B$ never gets in $\mathbb{D}$, in which case we set $\mu(z)=0$, or there is a first visit $B^{n}(z) \in \mathbb{D}$ with $n \in \mathbb{N}$, in which case we set $\mu(z)$ to be the value at $z$ of the pull-back of $\mu_{0}$ by $B^{n}$.

Now we straighten $\mu_{0}$ : There exists a quasiconformal homeomorphism $S: \mathbb{S}^{2} \rightarrow$ $\mathbb{S}^{2}$ that pushes $\mu_{0}$ to $0\left(S_{*} \mu_{0}=0\right)$, unique if we require that $S$ sends $\phi^{-1}(0)$ to 0 , $\infty$ to $\infty$, and 1 to $-\rho / 2$ where $\rho=e^{i 2 \pi \theta}$. The function

$$
F=S \circ \widetilde{B} \circ S^{-1}: \mathbb{S}^{2} \rightarrow \mathbb{S}^{2}
$$

is holomorphic, thus it is rational. The unique preimage of $\infty$ by $F$ is itself, thus $F$ is a polynomial. Its degree must coincide with its ramified covering degree, that is 2 .

The map $\phi \circ S^{-1}$ from $S(\mathbb{D})$ to $\mathbb{D}$ is holomorphic (the Beltrami form 0 is mapped to $\mu_{0}$ by $S^{-1}$ and then back to 0 by $\phi$ ), maps 0 to 0 , and conjugates $F$ to the rotation $R_{\theta}: \mathbb{D} \rightarrow \mathbb{D}$; thus $F$ fixes 0 with multiplier $e^{i 2 \pi \theta}$. Thus $F(z)=\rho z+a z^{2}$ for some $a \in \mathbb{C}^{*}$. On one hand this map has only one critical point $z=-\rho / 2 a$; on the other hand, $S$ sends the ramification point $z=1$ of $\widetilde{B}$ to $z=-\rho / 2$. Thus $a=1$ :

$$
F(z)=\rho z+z^{2} .
$$

This proves that $\widetilde{B}$ is a model for $F$.

\section{The GeOMETRIC CONSTRUCTION OF THE PRE-MODEL}

The Blaschke product $B$ is usually introduced by a formula. If we want a premodel for other families than the quadratic polynomials, it seems that we need to find the corresponding formulas. We present here a geometric construction of $B$ that does not require a formula, and that generalizes easily. We carry out this generalization for some entire maps and also for some Lavaurs maps. In each case, the pre-model will be called $\beta$.

3.1. For quadratic polynomials. Let $D$ be the open ball in $\mathbb{C}$ of center 1 and radius 1 .

\footnotetext{
${ }^{3}$ see section 4 for statements

${ }^{4}$ idem
} 


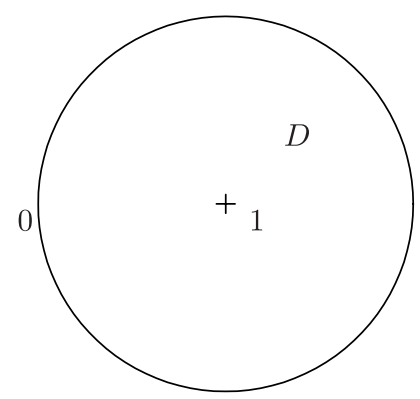

The preimage of $\partial D$ by the map $q: z \mapsto z^{2}$ is an $\infty$ shaped curve (a lemniscate).

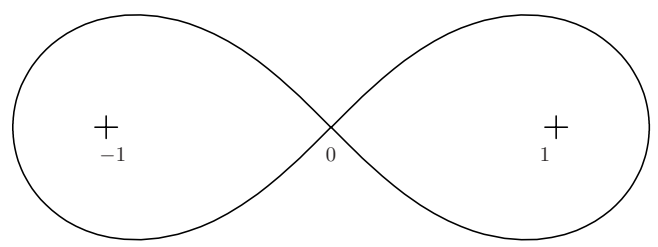

It cuts the complex plane into three connected components. The unbounded one maps 2-to-1 to $\mathbb{C} \backslash \bar{D}$ by $q$; the other two map isomorphically to $D$. Let us call $U$ the bounded one that contains $z=-1$, and let $W$ be the complement of $\bar{U}$ in $\mathbb{S}^{2}$.

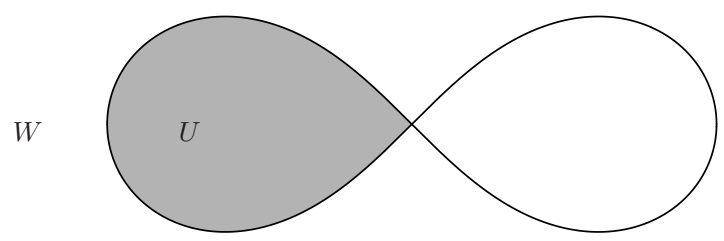

Let $\phi$ be the unique conformal isomorphism from $W$ to $\mathbb{S}^{2} \backslash \overline{\mathbb{D}}$ that fixes $\infty$ and maps 0 (the angular point of $U$ ) to 1 .

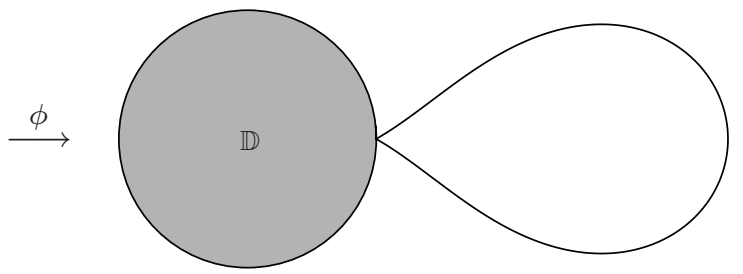

Let $b=t \circ q \circ \phi^{-1}: \mathbb{S}^{2} \backslash \overline{\mathbb{D}} \rightarrow \mathbb{S}^{2}$, where $t(z)=1-z$. Then $b(z)$ tends to $\partial \mathbb{D}$ when $z$ tends to $\partial \mathbb{D}$, thus $b$ admits a Schwarz reflection along $\mathbb{S}^{1}$ : That means there exists a holomorphic function $B: \mathbb{S}^{2} \rightarrow \mathbb{S}^{2}$ such that $B(z)=b(z)$ when $z \notin \overline{\mathbb{D}}$, and such that $B(z)=s(b(s(z)))$ when $z \in \mathbb{D}$, where $s(z)=1 / \bar{z}$. Moreover $B\left(\mathbb{S}^{1}\right) \subset \mathbb{S}^{1}$, thus $B$ is a Blaschke fraction. Every point not in $\mathbb{S}^{1} \cup\{0, \infty\}$ has 3 preimages by $B$, thus $B$ has degree 3 . It fixes $\infty$ with local degree 2 , thus it is of the form

$$
B(z)=\lambda z^{2} \frac{z-a}{1-\bar{a} z}
$$




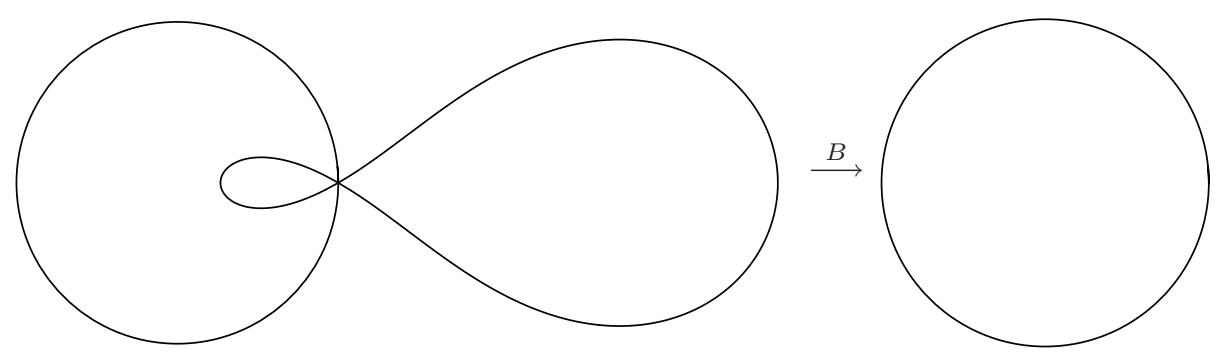

where $a \in \mathbb{C}^{*} \backslash \mathbb{S}^{1}$, and $\lambda \in \mathbb{S}^{1}$. The point $z=1$ is the unique preimage of 1 , thus $z=1$ is a local degree 3 critical point of $B$. This implies $a=3$. Finally, 1 is mapped to 1 , thus $\lambda=1$. We have proved that

$$
B(z)=z^{2} \frac{z-3}{1-3 z} \text {. }
$$

For this case, what we call the pre-model and denote by $\beta$ is just the function $B$.

3.2. For some entire maps. Let $\theta$ be a bounded type irrational and $\rho=\exp (i 2 \pi \theta)$. Let us give the class of entire maps we are able to study.

Definition 1. We assume $f: \mathbb{C} \rightarrow \mathbb{C}$ is holomorphic, fixes 0 with multiplier $\rho$, and has a set of singular values contained in $\{0,1\}$ and that $f$ is not linear 5 We make one more assumption: The connected component $U$ of $f^{-1}(\mathbb{D})$ containing 0 is bounded.

This last assumption is equivalent to the following: For any Jordan $\operatorname{arc} \gamma$ from 0 to 1 , the lift by $f$ of $\gamma$ starting from 0 does not tend to $\infty$. This implies that it tends to some point, independent of the choice of $\gamma$. This point is critical, otherwise $f$ would be linear. We will call it the main critical point.

Note that we allow polynomials. In this case, the last assumption of the definition is automatic.

These are very restrictive conditions. However, we will see that there are uncountably many topologically inequivalent such maps in section 7.

The restriction $f: U \backslash f^{-1}(0) \rightarrow \mathbb{D} \backslash\{0\}$ is a covering. Since $U$ contains 0 and $f$ has local degree 1 at this point, $f: U \rightarrow \mathbb{D}$ is an isomorphism. Moreover, $U$ is a Jordan disk (the bounded component of the complement of a Jordan curve), and $f: \bar{U} \rightarrow \overline{\mathbb{D}}$ is a homeomorphism. Let $z$ be the preimage of 1 by this homeomorphism. Let $\phi$ be the conformal isomorphism between $\mathbb{C} \backslash \bar{U}$ and $\mathbb{C} \backslash \overline{\mathbb{D}}$ whose extension to boundaries map $z$ back to 1 . Consider the map $f \circ \phi^{-1}: \mathbb{C} \backslash \overline{\mathbb{D}} \rightarrow \mathbb{C}$. It tends to $\partial \mathbb{D}$ when the variable tends to $\partial \mathbb{D}$, thus it has a Schwarz reflection extension $\beta: \mathbb{C} \backslash\{0\} \rightarrow \mathbb{C}$. The map $\beta$ is our pre-model.

3.3. For some Lavaurs maps. I have invented the geometric construction of premodels in my thesis [C], precisely because none were known for Lavaurs maps.

We will assume that the reader is familiar with the limit objects that arise in parabolic implosion. They were introduced, among others, by Douady, A. Epstein, Hubbard, Lavaurs and Shishikura. We give a small summary of notations and definitions in section 9 Some references are [C], D2], and [Sh].

${ }^{5}$ then, 1 is necessarily a singular value 
We consider the quadratic polynomial

$$
P(z)=v z+z^{2}
$$

where $v$ is a root of unity. Let us write

$$
v=\exp \left(i 2 \pi \frac{p}{q}\right)
$$

with $q \in \mathbb{N}^{*}, p \in \mathbb{Z}$, and $p / q$ irreducible. Let $K$ be the filled-in Julia set of $P$.

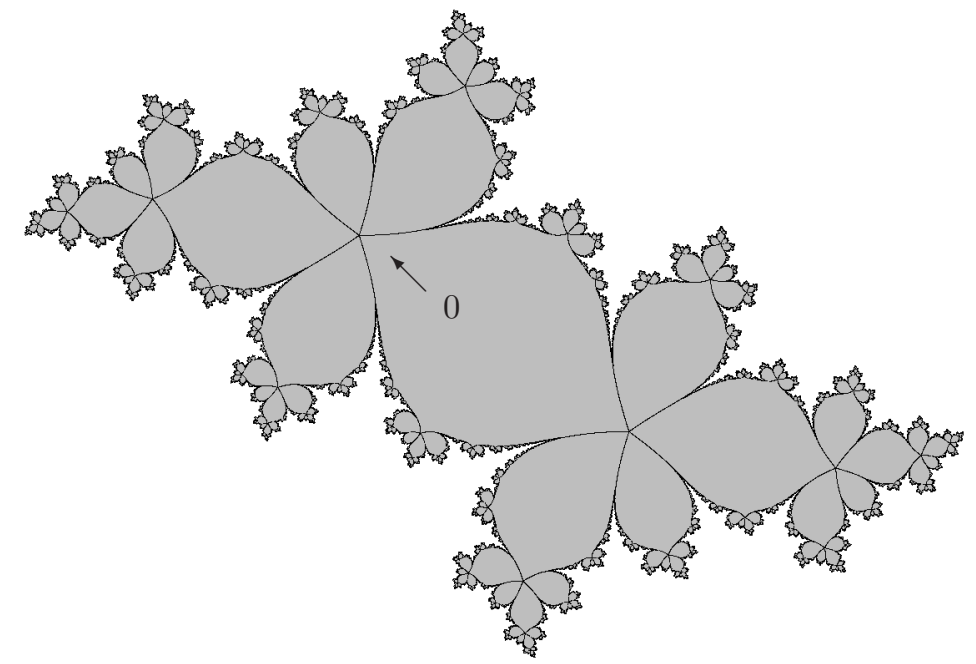

Figure 1. The filled-in Julia set of $P$ for $p / q=2 / 5$ (boundary in black, interior in gray).

Throughout this article, the notation $T_{a}$ refers to the translation by the vector $a$ on either the real line $\mathbb{R}$, the complex plane $\mathbb{C}$, or their quotient by the subgroup $\mathbb{Z}$.

The repelling axes of the parabolic point $z=0$ are labeled by $j$, which can take $q$ values. The attracting ones are labeled by $i$, which can also take $q$ values. The extended repelling Fatou parameterization associated to the $j$ th axis is denoted by

$$
\psi_{+, j}: \mathbb{C}_{j} \rightarrow \mathbb{C}
$$

where $\mathbb{C}_{j}$ is just a labeled copy of $\mathbb{C}$. It is a semi-conjugacy from $T_{1}$ to $P^{q}: \psi_{+, j} \circ$ $T_{1}=P^{q} \circ \psi_{+, j}$. The horn maps are maps

$$
h_{j, \sigma}: \psi_{+, j}^{-1}(\stackrel{\circ}{K}) \rightarrow \mathbb{C}_{j}
$$

where $\sigma$ is a complex parameter (see section 9 for more details). Their domain of definition does not depend on $\sigma$. It is invariant by $T_{1}$, and $h_{j, \sigma}$ commutes with the translation $T_{1}$. Thus there is a quotient map from a subset of the cylinder $\mathbb{C} / \mathbb{Z}$ to the cylinder, that we will denote by

$$
\bar{h}_{j, \sigma} .
$$

This quotient is defined in neighborhoods of the two ends of the cylinder, and has a holomorphic extension to these ends. This extension will be denoted by

$$
\widehat{h}_{j, \sigma} \text {. }
$$

It fixes both ends, with non-zero multiplier. 
For a fixed $\sigma$, the $q$ maps $\widehat{h}_{j, \sigma}$ with different $j$ are all conjugated by a translation. Thus from now on, we will fix an arbitrary $j$ and omit the index $j$ from the notations (because to study their dynamics, it is enough to work with any one of them).

Let $\theta$ be a bounded type irrational number. From the definition of the horn maps it follows that $h_{\sigma}=T_{\sigma} \circ h_{0}$. Thus there is a value of $\sigma$, unique modulo $\mathbb{Z}$, such that the upper end has multiplier $\exp (i 2 \pi \theta)$. The map $\widehat{h}_{\sigma}$ has thus a Siegel disk there, and we are looking for a model.

The construction of the pre-model does not depend on $\sigma$. So, for the rest of this section, we choose any value of $\sigma$, and write $h$ in place of $h_{\sigma}$. The map $\bar{h}$ has only one critical value $v$. It is an infinite degree ramified cover of the cylinder. Back to the complex plane, the preimage by $h$ of the horizontal line $L$ through $v$ is called the chessboard graph. The connected components of the complement in $\operatorname{Def}(h)$ of this preimage, are called the chessboard boxes. Each is mapped isomorphically by $h$ to either the upper or the lower half-plane delimited by the line $L$.
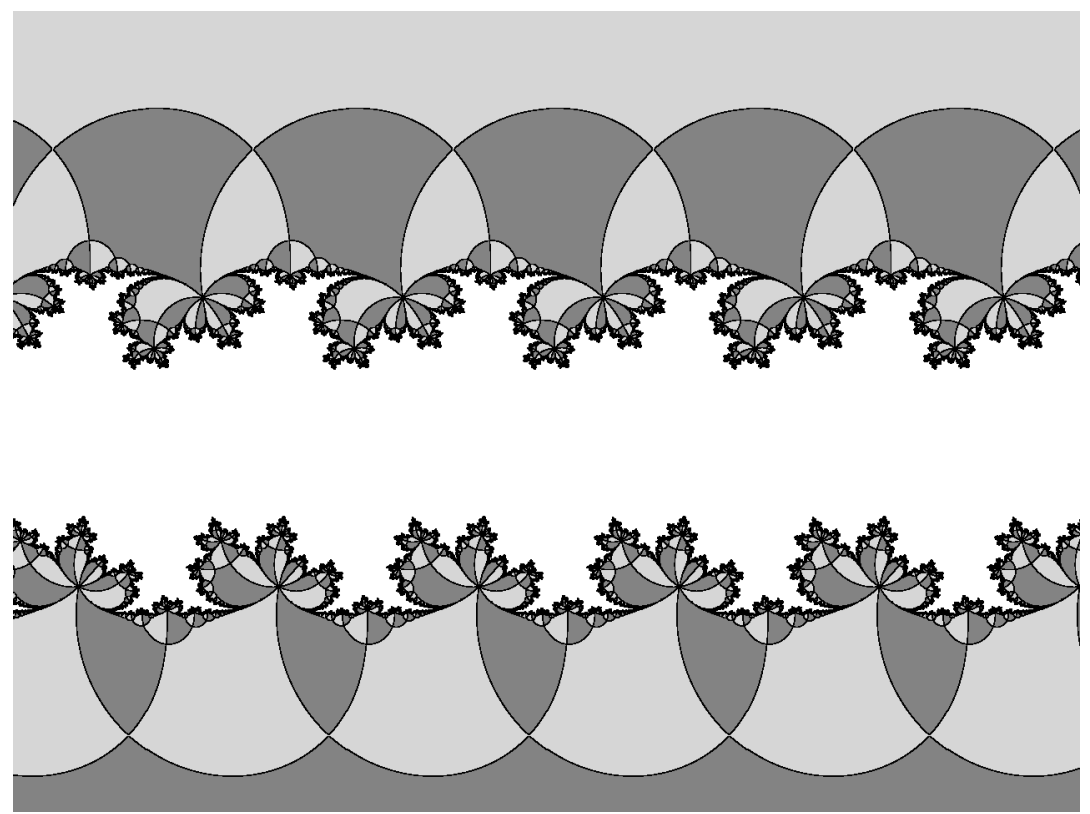

Figure 2. Parabolic chessboard of $h$ for $p / q=2 / 5$; the boxes that map to the upper half-plane are in light gray, those mapping to the lower half-plane in dark gray. The white set is the preimage by $\psi_{+}$of $\mathbb{C} \backslash K$. It is open and its closure is the complement of $\operatorname{Def}(h)$.

There is one chessboard box that is a neighborhood of the upper end of the cylinder. Let us call it $U$. Then $\partial U$ is a simple closed curve and $\bar{h}$ is a homeomorphism from the closure of $U$ to the upper half cylinder delimited by $L$. There is thus exactly one point $w$ on the boundary of $U$ that maps to $v$. Let $W$ be the 

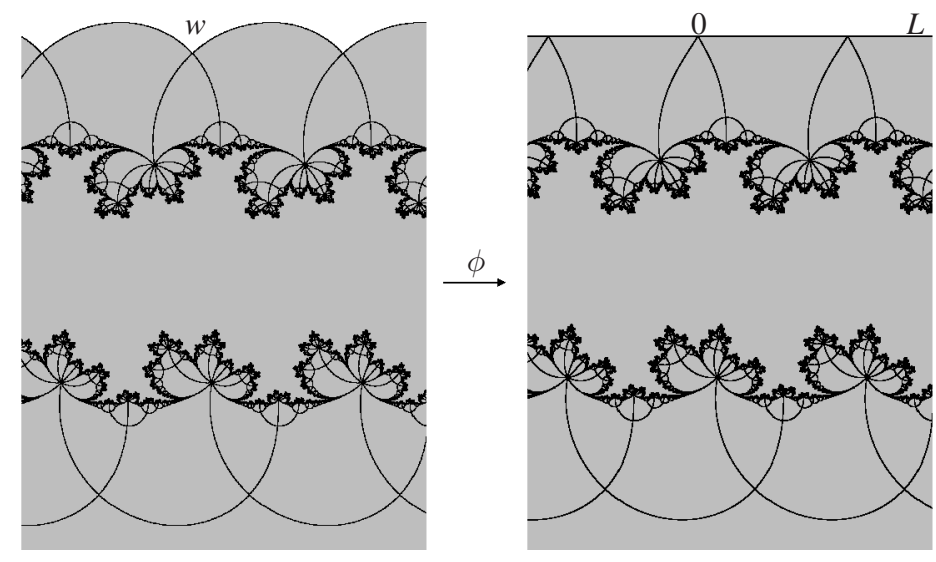

FiguRE 3 . The map $\phi$ is an isomorphism of the gray parts.
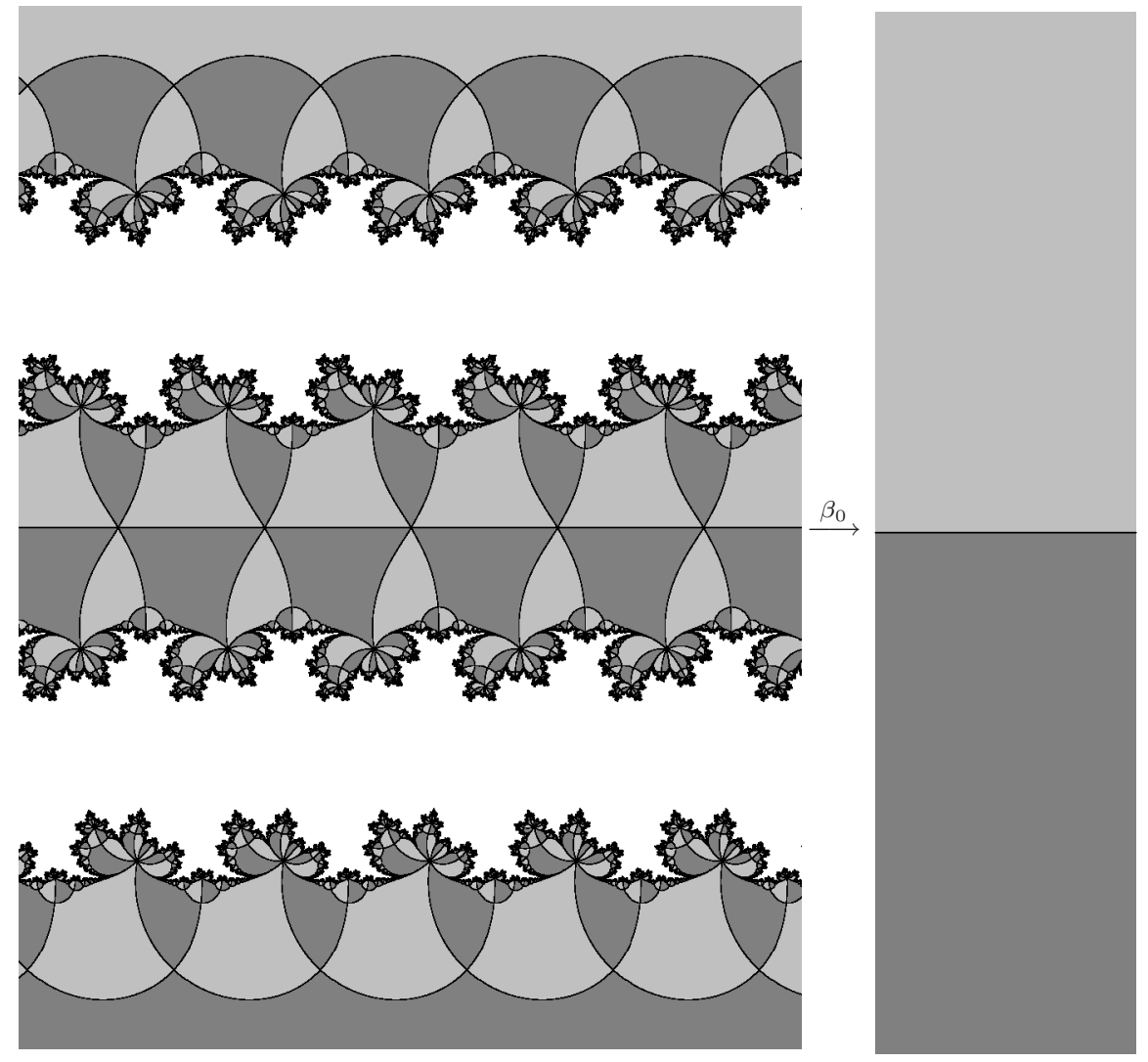
complement in the cylinder of the closure of $U$. Let $\phi$ be the unique analytic isomorphism between $W$ and the lower half cylinder delimited by the real line $\mathbb{R}$, that maps $w$ to 0 .

The map $T_{-v} \circ \bar{h} \circ \phi^{-1}$ has a Schwarz reflection $\beta_{0}$, because points tending to the real line have image tending to the real line. The map $\beta_{0}$ goes from a $T_{1}$-invariant subset of $\mathbb{C}$ to $\mathbb{C}$, and commutes with $T_{1}$. Its quotient $\beta$ is our pre-model: It goes from a subset of the cylinder $\mathbb{C} / \mathbb{Z}$ to $\mathbb{C} / \mathbb{Z}$. The map $\beta_{0}$ sends 0 to 0 , its set of real critical points is $\mathbb{Z}$, and its set of all critical values is $\mathbb{Z}$. Thus $\beta$ has only one critical value.

\section{From the PRE-MOdel to THE MODEL}

This section describes a standard argument due, among others, to Ghys, Douady, Shishikura, Herman and Świątek. In this article, quasiconformal, quasiregular and quasisymmetric maps will be always understood to be orientation preserving. We assume that the reader is familiar with these notions and with the straightening theorem (also called measurable Riemann mapping theorem) of Beltrami forms (also called almost-complex structures, or ellipsis fields). For an irrational $\theta$ with continued fraction expansion $\theta=a_{0}+1 /\left(a_{1}+1 /\left(a_{2}+\ldots\right)\right)$, let us call $\sup _{i>0} a_{i}$ the type of $\theta$.

Theorem 4.1 (Herman, Świątek). Suppose $f: \mathbb{T} \rightarrow \mathbb{T}$, where $\mathbb{T}=\mathbb{R} / \mathbb{Z}$ is an orientation preserving homeomorphism such that:

(1) $f$ has rotation number $\theta$, irrational of bounded type, bounded by $M \in \mathbb{N}^{*}$.

(2) $f$ is $C^{3}$ on $\mathbb{T}$.

(3) $f$ has a unique critical point, which is 0 .

(4) There is a neighborhood $W$ of 0 such that $f$ has negative Schwarzian derivative on $W \backslash\{0\}$.

(5) $\exists l \in \mathbb{N}^{*}$ and a real number $A>0$, such that $\forall x \in W, A|x|^{2 l} \leq f^{\prime}(x) \leq$ $2 A|x|^{2 l}$.

(6) The variation of $\log f^{\prime}$ on $\mathbb{T} \backslash W$ is bounded by $M^{\prime}>0$.

Then there is some $c>1$ such that $f$ is c-quasisymmetrically conjugate to the translation by $\theta$ on $\mathbb{T}$.

I borrowed this statement from [P2]. On the other hand, if $\theta$ does not have bounded type, then $f$ is never quasisymmetrically conjugate to the rotation $T_{\theta}$.

In the preceding section, we have constructed a pre-model map $\beta$, whose restriction to $\mathbb{S}^{1}$ (for entire maps) or $\mathbb{T}$ (for Lavaurs maps), is a homeomorphism. The quotient $\mathbb{T}$ is identified with the unit circle $\mathbb{S}^{1}$ in $\mathbb{C}$ by means of the map $t \mapsto \exp (i 2 \pi t)$. The cylinder $\mathbb{C} / \mathbb{Z}$ is identified with the subset $\mathbb{C}^{*}$ of the Riemann sphere $\mathbb{S}^{2}$, by means of the same formula. Let $\theta$ be an irrational. There is a unique real number $\tau$ such that $R_{\tau} \circ \beta$ has rotation number $\theta$ on $\mathbb{S}^{1}$ (see $\mathrm{dMvS}$ ). Suppose now $\theta$ has bounded type. The map $R_{\tau} \circ \beta$ satisfies the conditions of the Herman-Świątek theorem. Let $\eta$ be the quasisymmetric conjugacy given by this theorem. 
Theorem 4.2 (Ahlfors, Beurling). Every quasisymmetric map $f: \mathbb{S}^{1} \rightarrow \mathbb{S}^{1}$ extends to a quasiconformal map $\mathbb{S}^{2} \rightarrow \mathbb{S}^{2}$.

Douady and Earle enhanced this theorem in $\mathrm{DE}$.

Let $\widetilde{\eta}$ be such an extension of $\eta$ to $\mathbb{D}$. We will moreover require that $\widetilde{\eta}$ fix 0 , which is possible by post-composing any quasiconformal extension with a $C^{\infty}$ map equal to the identity in a neighborhood of the unit circle. Let $\mu_{0}$ be the pull-back of the null Beltrami form by $\widetilde{\eta}$ : $\mu_{0}$ is defined on $\mathbb{D}$. Let $\widetilde{\beta}=R_{\tau} \circ \beta$ outside of $\mathbb{D}$, and $\widetilde{\beta}=\widetilde{\eta}^{-1} \circ R_{\theta} \circ \widetilde{\eta}$. Then it is possible to define a globally $\widetilde{\beta}$-invariant Beltrami form $\mu$, by means of the dynamics. The well-known procedure goes as follows: If a point $z$ never falls in $\mathbb{D}$, let $\mu(z)=0$. Otherwise, let $z_{n}$ be the first iterate that falls in $\mathbb{D}$. Then define $\mu(z)$ to be the pull-back of $\mu_{0}\left(z_{n}\right)$ by $\widetilde{\beta}^{n}$.

The map $\widetilde{\beta}$ is called the model.

\section{Rigidity}

The notion of rigidity involved here is different from the usual notion of quasiconformal rigidity. In the classical rigidity theory one studies quasiconformal conjugacy classes of holomorphic maps. Here we focus on equivalence classes (which are simpler).

5.1. Entire maps. The following proposition is an isotopy lifting theorem (compare with [EL, lemma 2).

Proposition 5.1 (rigidity). Suppose $f$ and $g$ are two entire maps with a discrete set of singular value ${ }^{6}$, and $\phi$ and $\psi$ are two orientation-preserving homeomorphisms of $\mathbb{C}$, such that $\psi$ is isotopic to the identity rel. a set $V$ containing all the singular values of $f$, and such that the following diagram commutes:

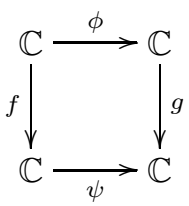

Then there exists a complex-affine function a (i.e. of the form $a(z)=b z+c$ ) such that the following diagram commutes

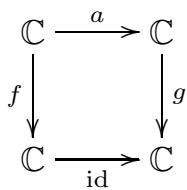

and such that $a$ is isotopic to $\phi$ rel. $f^{-1}(V)$. In particular, for all $z \in f^{-1}(V)$, $a(z)=\phi(z)$.

\footnotetext{
${ }^{6}$ Recall that the set of singular values is closed in $\mathbb{C}$, and that a discrete closed set of $\mathbb{C}$ has no accumulation points in $\mathbb{C}$.
} 
As an immediate corollary:

Corollary 5.1. If $f$ and $g$ are two entire maps with at most two singular values and if $f$ and $g$ are positively topologically equivalent (i.e. $\psi \circ f=g \circ \phi$ with $\psi$ and $\phi$ two orientation preserving homeomorphisms of $\mathbb{C}$ ), then they are affine equivalent (i.e. the same relation holds for a pair of complex affine maps $\phi$ and $\psi$ ). Moreover, let $V$ be a pair of distinct points containing the critical values of $f$ and let us write the topological equivalence and the affine one with the following respective commutative diagrams:
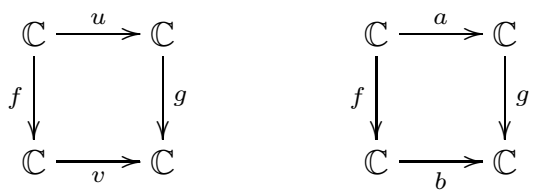

Then $a$ and $b$ can be chosen so that $v$ and $b$ take the same value on the set $V$, and such that $u$ and a take the same values on $f^{-1}(V)$.

Proof. Let $V=\left\{z_{0}, z_{1}\right\}$. Let $A$ be the unique affine map mapping $z_{0}$ to 0 and $z_{1}$ to 1. Let $w_{0}=v\left(z_{0}\right)$ and $w_{1}=v\left(z_{1}\right)$. Let $B$ be the unique affine map mapping $w_{0}$ to 0 and $w_{1}$ to 1 . Let $F=A \circ f$ and $G=B \circ g$. Then $F$ and $G$ satisfy the hypotheses of proposition 5.1, with $\phi=u, \psi=B \circ v \circ A^{-1}$ and $V^{\prime}=A(V)$ : Indeed, $\psi$ fixes 0 and 1 , and the only non-trivial hypothesis to check is that $\psi$ is isotopic to identity rel. $\{0,1\}$. A topological theorem states that every orientation preserving homeomorphism of $\mathbb{C}$ is isotopic to identity. Let $t \mapsto \psi_{t}$ be such an isotopy, with $t \in[0,1], \psi_{0}=\psi$ and $\psi_{1}=$ id. Let $C_{t}$ be the unique affine homeomorphism mapping $\psi_{t}(0)$ to 0 and $\psi_{t}(1)$ to 1 . Then $C_{t}$ varies continuously with $t$, and the map $t \mapsto C_{t} \circ \psi_{t}$ is an isotopy rel. $\{0,1\}$ from $\psi$ to id. So we can apply proposition 5.1 , and this tells us that there exists some affine map $a$ such that $F=G \circ a$, i.e. $A \circ f=B \circ g \circ a$, i.e. $b \circ f=g \circ a$ where $b=\left(B^{-1} \circ A\right)$. Proposition 5.1 also states that $a$ and $u$ have the same values on the set $F^{-1}\left(V^{\prime}\right)=f^{-1}(V)$. It is easy to check that $b$ and $v$ take the same values on $z_{0}$ and $z_{1}$.

5.2. Lavaurs maps. The difference here is that these maps are not rigid, not even in the cylinder. Take any homeomorphism $u: \mathbb{C} \rightarrow \mathbb{C}$ which is conformal on the domain of definition of the horn map $h$, commutes with $T_{1}$, but changes the $\mathbb{C}$-affine shape of $\operatorname{Def}(h)$. Then the map $h \circ u$ is equivalent (not conjugated) to $h$, but not affine equivalent.

To recover rigidity we need further assumptions on the maps in the equivalence, and we will make use of the quasiconformal conjugacy rigidity of the polynomial $P(z)=v z+z^{2}$.

Proposition 5.2 (folk). Let $v$ be a root of unity. Any holomorphic map $f: \mathbb{C} \rightarrow \mathbb{C}$ quasiconformally conjugated to $P(z)=v z+z^{2}$ is affine conjugated to $P$. Moreover, if $f=P$, then the conjugacy fixes any point in the grand orbit of the critical point, and any point in the Julia set $J$, and it leaves invariant each component of the complement of $J$. 
Remark. In fact, it is known that the quasiconformally rigid 7 quadratic polynomials are exactly those who belong to the boundary of the Mandelbrot set, and the above proposition holds for them.

Consider now a Beltrami form $\mu$ that is the pull-back by $\psi_{+}$of some Beltrami form $\mu^{\prime}$ on $\mathbb{C}$. Then $\mu$ is $T_{1}$-invariant if and only if $\mu^{\prime}$ is $P^{q}$-invariant.

Proposition 5.3. Let $P$ be as in the preceding proposition. Let $\mu$ be a Beltrami form defined on $\mathbb{C}$ such that:

- $\mu$ is the pull-back by $\psi_{+}$of some P-invariant Beltrami form $\mu^{\prime}$ on $\mathbb{C}$.

- $\|\mu\|_{\infty}<1$.

Let $J$ be the Julia set of $P$. Let $J^{\prime}=J \cup\{$ the grand orbit of the critical point of $P\}$. Let $w_{0}$ be any point in $\psi_{+}^{-1}\left(J^{\prime}\right)$. Let $s$ be the unique straightening of $\mu$ which commutes with $T_{1}$ and fixes $w_{0}$. Then $s$ fixes every point in $\psi_{+}^{-1}\left(J^{\prime}\right)$, and leaves invariant every component of the complement of $\psi_{+}^{-1}(J)$.

Note that the hypotheses imply that $\mu=\lambda \mu_{0}$ on the complement of $\operatorname{Def}(h)$, where $\lambda \in \mathbb{C},|\lambda|<1$ and $\mu_{0}$ is the pull-back by $\psi_{+}$of the line field parallel to external rays of the Julia set. There is much more freedom in $\operatorname{Def}(h)$ (the vector space of all such $\mu$ 's is infinite dimensional). Note also that we not only require $\mu^{\prime}$ to be $P^{q}$-invariant, but also $P$-invariant.

Proof. We only give an idea of the proof. According to proposition 5.2, there is a straightening $R$ of $\mu^{\prime}$ that conjugates $P$ to itself. We claim that there is a map $s: \mathbb{C} \rightarrow \mathbb{C}$ such that $\psi_{+} \circ s=R \circ \psi_{+}$. To prove this, one considers the set $\mathcal{B}$ of backward orbits of $P^{q}$ (sequences $\left(z_{n}\right)_{n \in \mathbb{N}}$ with $P^{q}\left(z_{n+1}\right)=z_{n}$ ) that tend to 0 along the repelling axis of index $j$ (the index that we fixed in section 9). The map that sends $w$ to the sequence $\psi_{+}(w-n)$ is a bijection from $\mathbb{C}$ to $\mathcal{B}$. Moreover, $R$ acts on $\mathcal{B}$, from which the claim follows. The same can be done for $R^{-1}$, which provides an inverse for $s$. One then proves that $s$ and $s^{-1}$ are continuous, thus $s$ is a homeomorphism. The relation $\psi_{+} \circ s=R \circ \psi_{+}$implies that $s$ is quasiconformal and maps $\psi_{+}^{*}\left(\mu^{\prime}\right)=\mu$ to $\psi_{+}^{*}(0)=0$ : In other words, it straightens $\mu$. Finally, $R$ leaves invariant any element $\left(z_{n}\right) \in \mathcal{B}$ such that $z_{0} \in J^{\prime}$, and the last statement of the proposition follows.

The proof yielded the following:

Proposition 5.4. Let $R$ be the straightening of $\mu^{\prime}$ that conjugates $P$ to itself. Then

$$
\psi_{+} \circ S=R \circ \psi_{+} .
$$

We will only make use of one implication of the next proposition, but we think it is worth mentioning the equivalence.

\footnotetext{
${ }^{7}$ rigidity with respect to quasiconformal conjugacy

${ }^{8}$ For this, let $V$ be a small disk centered on 0 , on which $P^{q}$ has an inverse branch. Every element of $\left(z_{n}\right) \in \mathcal{B}$ has an index $N$ for which $\forall n \geq N, z_{n} \in V$. Let $w_{k}$ be a sequence in $\mathbb{C}$, and $z_{n}^{k}=\psi_{+}\left(w_{k}-n\right)$. Let $w \in \mathbb{C}$ and $z_{n}=\psi_{+}(w-n)$. Then it is enough to notice that $w_{k}$ tends to $w$ if and only if:

1. there exists a common index $N$ for the sequences $z_{n}$ and $z_{n}^{k}$;

2. $z_{N}^{k} \longrightarrow z_{N}$ as $k \rightarrow+\infty$.
} 
Proposition 5.5. For a Beltrami form $\mu$ on $\mathbb{C}$ that is equal to 0 on $\mathbb{C} \backslash \operatorname{Def}(h)$, the condition that $\mu$ be a pull-back by $\psi_{+}$of a P-invariant Beltrami form is equivalent to the restriction of $\mu$ to $\operatorname{Def}(h)$ being a pull-back by $h$ of a $T_{1}$-invariant Beltrami form.

Proof. The map $h$ has the form $h=T_{\text {something }} \circ \phi_{\div} \circ \psi_{+}$(see section 9 for the definition of $\phi_{\div}$), so the proposition amounts to the following: The Beltrami forms $\mu^{\prime}$ on $\stackrel{\circ}{K}$ that are $P$-invariant are exactly the pull-backs by $\phi \div$ of $T_{1}$-invariant forms. This claim follows from the remark that two points $z$ and $z^{\prime}$ belong to the same grand-orbit by $P$ if and only if $\phi_{\doteqdot}(z)=\phi_{\doteqdot}(z) \bmod \mathbb{Z}$.

Proposition 5.6 (rigidity). Suppos $₫ 9 l: \operatorname{Def}(l) \rightarrow \mathbb{C}$ is holomorphic with $\operatorname{Def}(l) \subset$ $\mathbb{C}$. Suppose $u$ and $v$ are quasiconformal homeomorphisms of $\mathbb{C}$, which both commute with $T_{1}$. Suppose further that $u$ fixes at least one point $w_{0}$ in $\psi_{+}^{-1}\left(J^{\prime}\right)$ (where $J^{\prime}=J \cup$ the grand orbit of the critical point of $\left.P\right)$, that $\bar{\partial} u=0$ on $\mathbb{C} \backslash \operatorname{Def}(h)$, that $u(\operatorname{Def}(h))=\operatorname{Def}(l)$, and that the following diagram commutes:

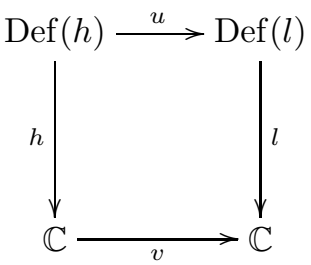

Then $\operatorname{Def}(l)=\operatorname{Def}(h)$ and there exists $\kappa \in \mathbb{C}$ such that

$$
l=T_{\kappa} \circ h .
$$

Proof. First, the hypotheses imply that there is a $\kappa \in \mathbb{C}$ such that $v$ coincides with $T_{\kappa}$ on the set of critical values of $h$. Let $\mu^{\prime}=u^{*}(0)$, and $\mu=v^{*}(0)$. The Beltrami form $\mu^{\prime}$ is zero on $\mathbb{C} \backslash \operatorname{Def}(h)$ and, on $\operatorname{Def}(h)$, it is the pull-back by $h$ of $\mu$. Thus according to proposition 5.5. proposition 5.3 applies to $\mu^{\prime}$. In particular, $u$ preserves each component $U$ of $\operatorname{Def}(h)$. Now, for $t \in[0,1]$, let $v_{t}$ be the straightening of $t \mu$ that coincides with $v$ on the set of critical values of $h: v_{1}=v$ and $v_{0}=T_{\kappa}$. Let $\pi: \mathbb{C} \rightarrow \mathbb{C} / \mathbb{Z}$ be the canonical quotient map. Since $\pi \circ h$ is a ramified cover (of infinite degree), with only one critical value, an isotopy lifting argument, like in the preceding section, shows that the isotopy $v_{t}$ can be pulled-back through the diagram to an isotopy $u_{t}$ on $\operatorname{Def}(h)$ such that $l \circ u_{t}=v_{t} \circ h, u_{t}$ commutes with $T_{1}$, and $u_{1}=\left.u\right|_{\operatorname{Def}(h)}$. The map $u_{0}$ is an analytic isomorphism of each component $U$ of $\operatorname{Def}(h)$. Since $u_{t}$ maps critical points $z$ of $h$ to critical points of $l$, the map $t \mapsto u_{t}(z)$ is constant on the critical points of $h$. Thus $u_{0}(z)=u_{1}(z)=u(z)=z$ for all critical points $z$ of $h$. Now, each component of $\operatorname{Def}(h)$ contains at least 2 critical points of $h$ (in fact, infinitely many), and has hyperbolic universal covering (a component omits more than two points in $\mathbb{C}$ ), thus $u_{0}$ is the identity on $\operatorname{Def}(h)$. This proves the proposition.

\footnotetext{
${ }^{9}$ For the reader that skipped directly to this proposition, $P(z)=v z+z^{2}$ is a quadratic polynomial with a parabolic fixed point, $J$ is its Julia set, $h$ its horn map, and $\Psi_{+}$a repelling Fatou coordinate.
} 


\section{The STRAightening}

Here, we prove that straightening the Beltrami form provides the expected map.

6.1. Entire maps. Let us consider a map $f$ as in section 3.2 Let $\beta$ be the associated pre-model. Let $\theta$ be any bounded type irrational and $\widetilde{\beta}$ the model defined in section 4. A conformal isomorphism $\phi: \mathbb{C} \backslash \bar{U} \rightarrow \mathbb{C} \backslash \overline{\mathbb{D}}$ was defined, where $U$ is the connected component of $f^{-1}(\mathbb{D})$ that contains 0 . There was also a homeomorphism $\widetilde{\eta}$ of $\overline{\mathbb{D}}$, fixing 0 and quasiconformal on $\mathbb{D}$. The map $\widetilde{\beta}$ was defined by

$$
\widetilde{\beta}=R_{\tau} \circ f \circ \phi^{-1}
$$

on $\mathbb{C} \backslash \overline{\mathbb{D}}$ and

$$
\widetilde{\beta}=\widetilde{\eta}^{-1} \circ R_{\theta} \circ \widetilde{\eta}
$$

on $\overline{\mathbb{D}}$. Let us extend $\phi$ into a (quasiconformal) homeomorphism $\widetilde{\phi}$ of $\mathbb{C}$, by means of the formula

$$
\widetilde{\phi}=\widetilde{\eta}^{-1} \circ R_{-\theta} \circ \widetilde{\eta} \circ R_{\tau} \circ f
$$

on $\bar{U}$, with the notations of section 4. This extension has been chosen so that the relation

$$
\widetilde{\beta} \circ \widetilde{\phi}=R_{\tau} \circ f
$$

holds on all of $\mathbb{C}$. Thus $\widetilde{\beta}$ and $f$ are topologically equivalent. Let $\mu$ the $\widetilde{\beta}$-invariant Beltrami form defined in section $4 S$ the unique straightening (sending $\mu$ to 0 ) that maps 0 to 0 and that maps the unique non-zero singular value of $\widetilde{\beta}$, namely $R_{\tau}(1)$, to 1 , and $g$ the conjugated function

$$
g=S \circ \widetilde{\beta} \circ S^{-1} .
$$

It is holomorphic, entire, and topologically equivalent to $f$ with the following equivalence,

$$
g \circ(S \circ \widetilde{\phi})=\left(S \circ R_{\tau}\right) \circ f .
$$

According to corollary 5.1 $g \circ a=b \circ f$ with two affine isomorphisms $a$ and $b$, and with $b$ taking on 0 and 1 the same value as $S \circ R_{\tau}$, i.e. 0 and 1 , thus $b=\mathrm{id}$, and with $a$ taking the same value as $S \circ \widetilde{\phi}$ at every point of $f^{-1}(\{0,1\})$. Thus $a(0)=S \circ \widetilde{\phi}(0)=0$, which implies $a$ is of the form $z \mapsto \lambda z$ for some $\lambda \in \mathbb{C}^{*}$. Equivalence implies $g(\lambda z)=f(z)$, thus $\lambda g^{\prime}(0)=f^{\prime}(0)$. But near $0, g$ is conjugated to $R_{\theta}$ by the map $\widetilde{\eta} \circ S^{-1}$. This conjugacy is conformal because the pull-back of the null Beltrami form by $\widetilde{\eta}$ is $\mu$ and $S$ straightens $\mu$. Thus $g^{\prime}(0)=\exp (i 2 \pi \theta)=f^{\prime}(0)$, which implies $\lambda=1$. This proves that

$$
g=f
$$

which means that the model $\widetilde{\beta}$ is quasiconformally conjugated to $f$. 
Corollary 6.1. For all entire maps satisfying the conditions of section 3.2 , there is a Siegel disk containing 0 , whose boundary is a quasicircle containing exactly one critical point.

This critical point must be the main critical point, defined in section 3.2

6.2. Lavaurs maps. Let us sum up what we have obtained up to now, concerning Lavaurs maps. We had a root of unity $v$, the polynomial $P=v z+z^{2}$, we chose one of the repelling axes $j$, and denoted by $\psi_{+}$the repelling Fatou coordinate from $\mathbb{C}_{j}$ to $\mathbb{C}$. We constructed a pre-model map $\beta$. We were then given a bounded type irrational $\theta$ and we associated to it a model map $\widetilde{\beta}$. These objects (sets, maps, Beltrami form) live in the cylinder $\mathbb{C} / \mathbb{Z}$, and we now want to consider their lifts to the universal cover $\mathbb{C}$. But for convenience, we will use the same notations. We constructed a $\widetilde{\beta}$-invariant and $T_{1}$-invariant Beltrami form $\mu$. Let $w_{0}$ be any point in $\psi_{+}^{-1}(J)$. Straightening $\mu$ conjugates $\widetilde{\beta}$ to a holomorphic map $l$ :

$$
l=S \circ \widetilde{\beta} \circ S^{-1}
$$

where $S$ is the unique straightening of $\mu$ that commutes with $T_{1}$ and sends $\phi\left(w_{0}\right)$ to $w_{0}$ (the map $\phi$ is defined is section 3.3. the condition on $w_{0}$ is here to ensure that the domain of definition of $l$ coincides with that of $h$, as we will prove in a few paragraphs). The following theorem states that we have obtained what we were looking for:

\section{Theorem 6.1.}

$$
\exists \sigma \in \mathbb{C}: l=h_{\sigma}
$$

and

$$
\left(\widehat{h}_{\sigma}\right)^{\prime}(+i \infty)=\exp (i 2 \pi \theta) .
$$

So, $\widetilde{\beta}$ is a quasiconformal model for the horn map with a Siegel disk with rotation number $\theta$ at the upper end of the cylinder.

Proof of Theorem [6.1. We want to apply propositions [5.3 and [5.6. The map $\widetilde{\beta}$ and the Beltrami form $\mu$ are not well suited, because their domain of definition does not coincide with that of $h$. So we will consider another quasiconformal map $H$, quasiconformally conjugated to $\widetilde{\beta}$, thus preserving another Beltrami form $\mu^{\prime}$. We had called $U$ the upper chessboard box, and $\phi$ was an analytic isomorphism from $\mathbb{C} \backslash \bar{U}$ to $\mathbb{C} \backslash \overline{\mathbb{H}}$, that commutes with $T_{1}$. The map $\widetilde{\beta}$ is equal to $T_{\tau} \circ T_{-v} \circ h \circ \phi^{-1}$ on $\mathbb{C} \backslash \overline{\mathbb{H}}$, and to some quasiconformal "rotation" on $\overline{\mathbb{H}}$. So the domain of definition of $\widetilde{\beta}$ is $\overline{\mathbb{H}} \cup \phi(\operatorname{Def}(h))$. Recall that $h$ is a horn map whose phase we do not care about. The map $T_{\tau} \circ T_{-v} \circ h$ is also a horn map, and for convenience, from now on, $h$ will refer to that one. We want to conjugate $\widetilde{\beta}$ back by $\phi$. So we must first extend $\phi$ on $\bar{U}$ into a quasiconformal homeomorphism $\widetilde{\phi}$ of $\mathbb{C}$, and will then define

$$
H=\widetilde{\phi}^{-1} \circ \widetilde{\beta} \circ \widetilde{\phi}
$$

and the following $H$-invariant and $T_{1}$-invariant Beltrami form

$$
\mu^{\prime}=\widetilde{\phi}^{*}(\mu) \text {. }
$$

We do not have much choice on the definition $\widetilde{\phi}$, because of the requirement in propositions $5.3+5.5$ that $\mu^{\prime}$ be the pull-back by $h$ of some Beltrami form and be $T_{1}$-invariant. 
Lemma 6.1. There is one and only one extension $\widetilde{\phi}$ of $\phi$ such that $\mu^{\prime}$ is a pull-back by $h$, and it has the form

$$
\left.\widetilde{\phi}\right|_{\bar{U}}=\left(\left.\widetilde{\beta}\right|_{\overline{\mathbb{H}}}\right)^{-1} \circ h
$$

This is also the only extension such that $H$ is "compatible" with h, i.e. has the form $H=$ something oh. Moreover, the following holds on all of $\operatorname{Def}(h)$ :

$$
H=\widetilde{\phi}^{-1} \circ h
$$

Proof. Indeed, let $\widetilde{\phi}$ be any extension of $\phi$ such that the Beltrami form $\mu^{\prime}=\widetilde{\phi}^{*} \mu$ is a pull-back by $h$. Note that $\mu^{\prime}=\phi^{*} \mu$ on $\operatorname{Def}(h) \backslash U$. Now, the chessboard boxes of $h$ are mapped isomorphically by $h$ to half-planes delimited by $\mathbb{R}$. There are infinitely many mapped to each one, so there is certainly one mapped to the upper half-plane and different from $U$. Let us call it $V$. The form $\mu^{\prime}$ is uniquely determined on $V$, and it is pushed by the branch $\left.h\right|_{V}$ to a uniquely determined form on $\mathbb{H}$. The pull-back by the branch $\left.h\right|_{U}$ is thus uniquely determined: it means $\mu^{\prime}$ is unique. Thus any $\widetilde{\phi}$ has fixed Beltrami differential on $U$ and since it extends $\phi$, it has fixed values on the boundary of $U$, thus it is unique.

Let us now consider the compatibility requirement. Assume that a function $\widetilde{\phi}$ was defined such that the map $H$ defined by $H=\widetilde{\phi}^{-1} \circ \widetilde{\beta} \circ \widetilde{\phi}$ has the form $H=\operatorname{sg} \circ h$. Then, $\mu^{\prime}=\widetilde{\phi}^{*}(\mu)$ being $H$-invariant, it is certainly a pull-back by $h$. Since $H=\widetilde{\phi}^{-1} \circ \widetilde{\beta} \circ \widetilde{\phi}$ on $\mathbb{C}$ and $\widetilde{\beta}=h \circ \phi^{-1}$ on $\mathbb{C} \backslash \mathbb{H}$, we have $H=\widetilde{\phi}^{-1} \circ h$ on $\mathbb{C} \backslash \mathbb{H}$. But we know that $h(\mathbb{C} \backslash \mathbb{H})$ equals all of $\mathbb{C}$. The requirement $H=\operatorname{sg} \circ h$ on $\mathbb{C}$ thus implies that $\operatorname{sg}=\widetilde{\phi}^{-1}$ on all of $\mathbb{C}$. Thus $\widetilde{\phi}^{-1} \circ \widetilde{\beta} \circ \widetilde{\phi}=H=\widetilde{\phi}^{-1} \circ h$ on all of $\mathbb{C}$, which simplifies into $\widetilde{\beta} \circ \widetilde{\phi}=h$. Taken on $\bar{U}$, this yields the formula $\left.\widetilde{\phi}\right|_{\bar{U}}=\left(\left.\widetilde{\beta}\right|_{\mathbb{H}}\right)^{-1} \circ h$.

Conversely, one checks that this formula works: In other words, this $\widetilde{\phi}$ extends $\phi$ to a quasiconformal homeomorphism of $\mathbb{C}$ commuting with $T_{1}$, such that the relation $H=\widetilde{\phi}^{-1} \circ h$ holds on all of $\operatorname{Def}(h)$, and thus the $H$-invariant Beltrami form $\mu^{\prime}$ is a pull-back by $h$.

Let $\widetilde{\phi}$ be this extension. Let $s=S \circ \widetilde{\phi}$ be the conjugacy from $H$ to $l$. The map $s$ is quasiconformal, commutes with $T_{1}$, and fixes $w_{0}$. We have the following commutative diagram:

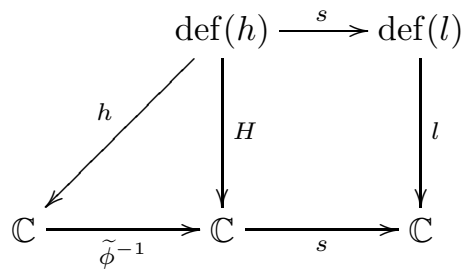


The outer part can be read:

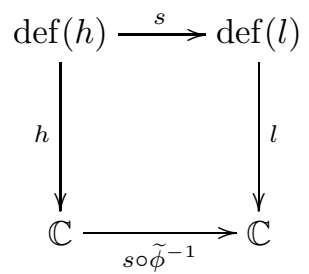

We now apply proposition 5.6 to it. This proves theorem 6.1

Thus $s(\operatorname{def}(h))=\operatorname{def}(l)=\operatorname{def}(h)$. As noted in the proof of proposition [5.6, $s$ satisfies the conditions of proposition 5.3 (the Beltrami form straightened by $s$ is $\left.\mu^{\prime}\right)$. Thus $s$ fixes every point in $\psi_{+}^{-1}\left(J^{\prime}\right)$.

Corollary 6.2. The Siegel disk $\Delta$ of the map $\widehat{h}_{\sigma}$ is compactly contained in the domain of definition of $\widehat{h}_{\sigma}$. Its boundary is a quasicircle, which contains the upper main critical point of $\widehat{h}_{\sigma}$ (the one which is on the boundary of the uppermost chessboard box), and no other.

Let us also use the notation $\Delta$ for the preimage of $\Delta$ by the covering map $\mathbb{C} \rightarrow \mathbb{C} / \mathbb{Z}$. All chessboard boxes $V$ are mapped by $h$ to the upper or the lower half-plane. Let us call $V$ light in the first case, and dark in the second case.

Corollary 6.3 (combinatorial description). The image of the light chessboard boxes of $\operatorname{def}(h)$ by the quasiconformal isomorphism s consists of the connected components of the first preimage by $h_{\sigma}$ of the Siegel disk $\Delta$. From each of these components, $h_{\sigma}$ is an isomorphism to $\Delta$. All the critical points of $h$ belong to the image by $s$ of the chessboard graph (the analytic lines separating the boxes). The dark boxes map by s to components mapped isomorphically to $\mathbb{C} \backslash \bar{\Delta}$ by $h_{\sigma}$.

So far, we analyzed the dynamics of the horn map $h_{\sigma}$, which live in the Fatou coordinates (more precisely the universal cover of the Ecalle-Voronin cylinders). Let us give an informal description of what happens in the dynamical plane where $P$ lives. A more precise version of this analysis is given in my thesis [C]. We saw that $\mu^{\prime}$ is the pull-back by $\psi_{+}$of some Beltrami form $\mu^{\prime \prime}$. Let us have a closer look at the proposition 5.4. The form $\mu^{\prime \prime}$ has a straightening $R$ preserving $P$, every point of $J$, every component of $\mathbb{C} \backslash J$, and every point in the grand orbit of the critical point. The map $R$ satisfies the relation $R \circ \psi_{+}=\psi_{+} \circ s$. It sends $\Delta$ to what is called the Virtual Siegel disk $\Delta^{\prime}$ of the (modified) Lavaurs map $g_{\sigma}$. It is periodic of period $q$ under $P$, and fixed by $g_{\sigma}$. We recall that $g_{\sigma}: \stackrel{\circ}{K} \rightarrow \mathbb{C}$ is holomorphic and satisfies the relation $g_{\sigma} \circ \psi_{+}=\psi_{+} \circ h_{\sigma}$. The filled-in Julia-Lavaurs set $K\left(P, g_{\sigma}\right)$ is by definition the complement of the escaping set, the latter being the union for $n \in \mathbb{N}$ of $g_{\sigma}^{-n}(\mathbb{C} \backslash K)$ for $n \in \mathbb{N}$. The Julia-Lavaurs set $J\left(P, g_{\sigma}\right)$ is by definition the boundary of $K\left(P, g_{\sigma}\right)$ in $\mathbb{C}$ (see [D2] and [L for details). The map $R$ maps the chessboard graph of $\stackrel{\circ}{K}$ into a subset of the Julia-Lavaurs set $J\left(P, g_{\sigma}\right)$, and every light box to a component of the first preimage of $\Delta^{\prime}$ by $g_{\sigma}$, or equivalently to an iterated preimage by $P$ of $\Delta^{\prime}$. The following pictures illustrate all this.

6.3. Illustrations. We illustrate in the following pages the case of the Lavaurs maps. 

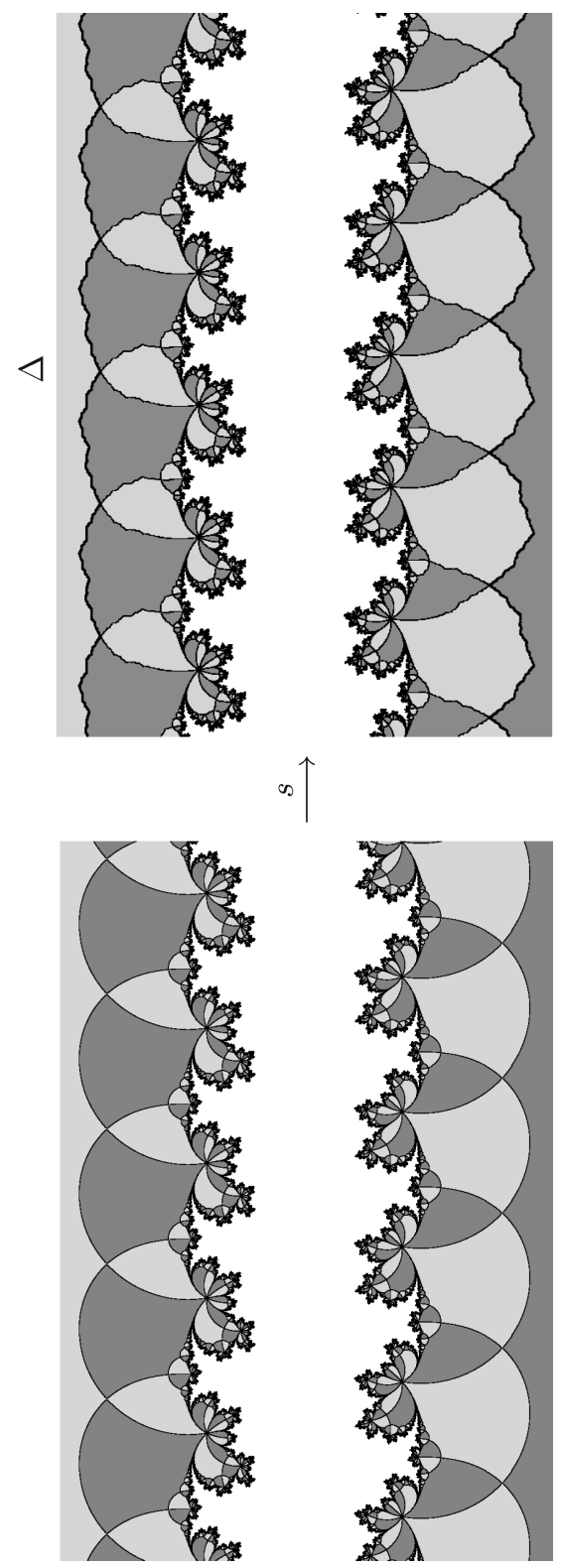

Figure 4. The parabolic chessboard in Fatou coordinates, and its image by the straightening $s$, for $p / q=2 / 5$, and $\theta=\frac{\sqrt{5}-1}{2}$ (the golden mean). The Siegel disk is at the upper end. The figure has been rotated by 90 degrees. 


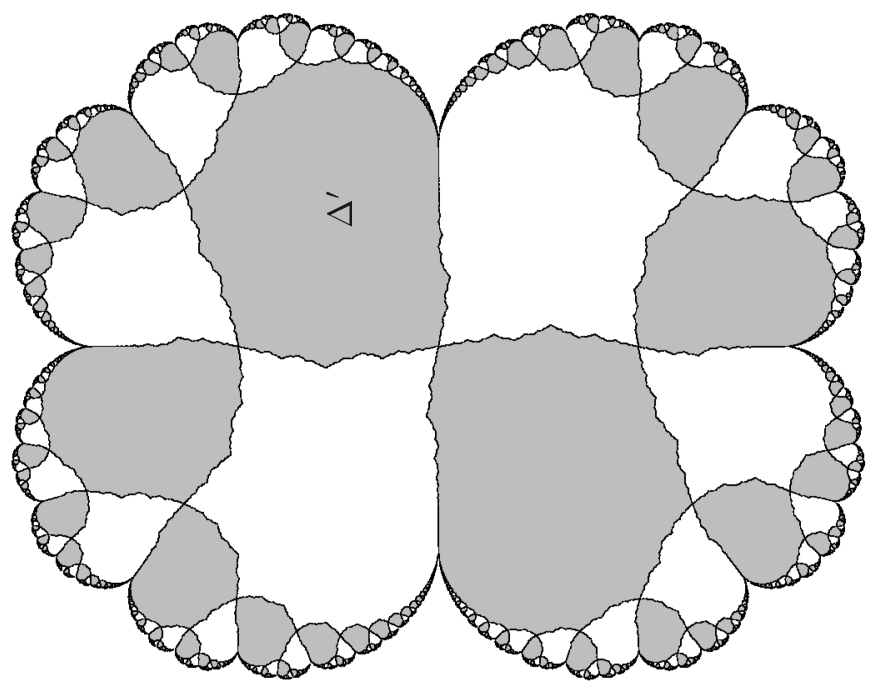

$2 \uparrow$

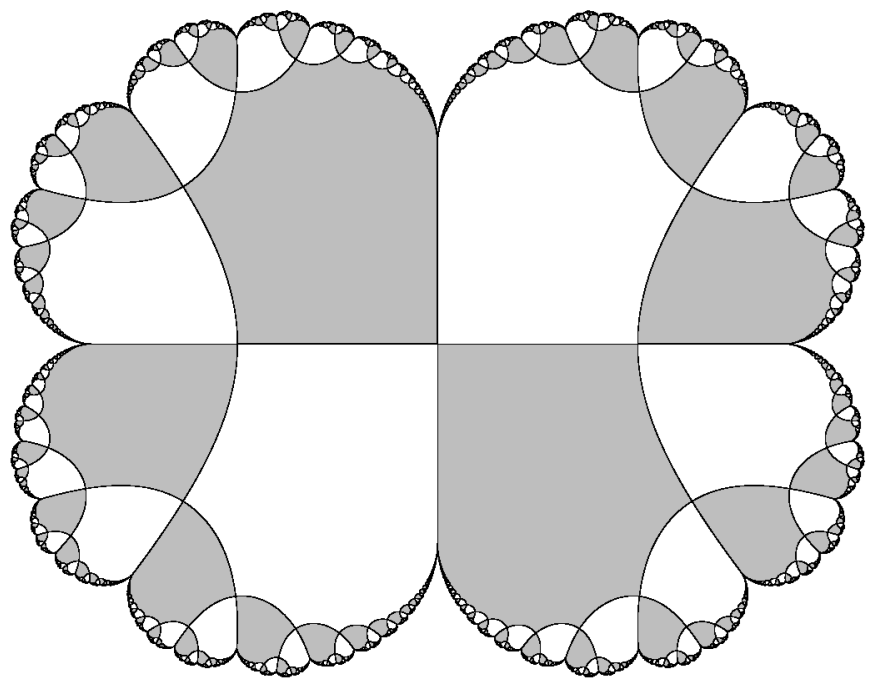

Figure 5. The parabolic chessboard in initial coordinates, for $p / q=0 / 1$, and its image by the straightening $R$, with $\theta=$ the golden mean. The figure has been rotated by 90 degrees. 


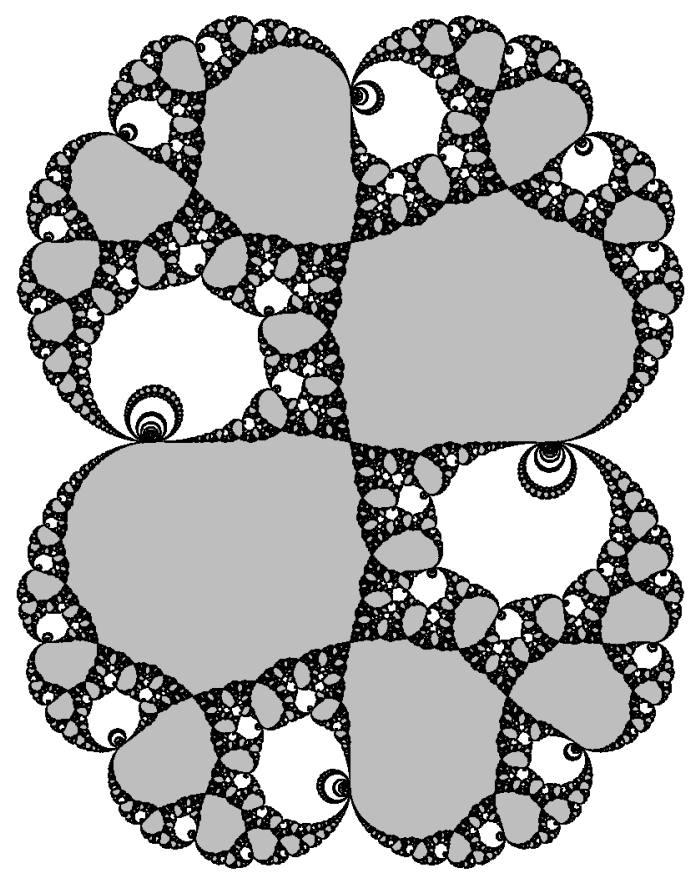

Figure 6 . The corresponding filled-in Julia-Lavaurs set. It is the non-escaping set under $P$ and $g_{\sigma}$ (with the appropriate value of $\sigma)$. The picture shows the boundary in black, and the interior in gray.

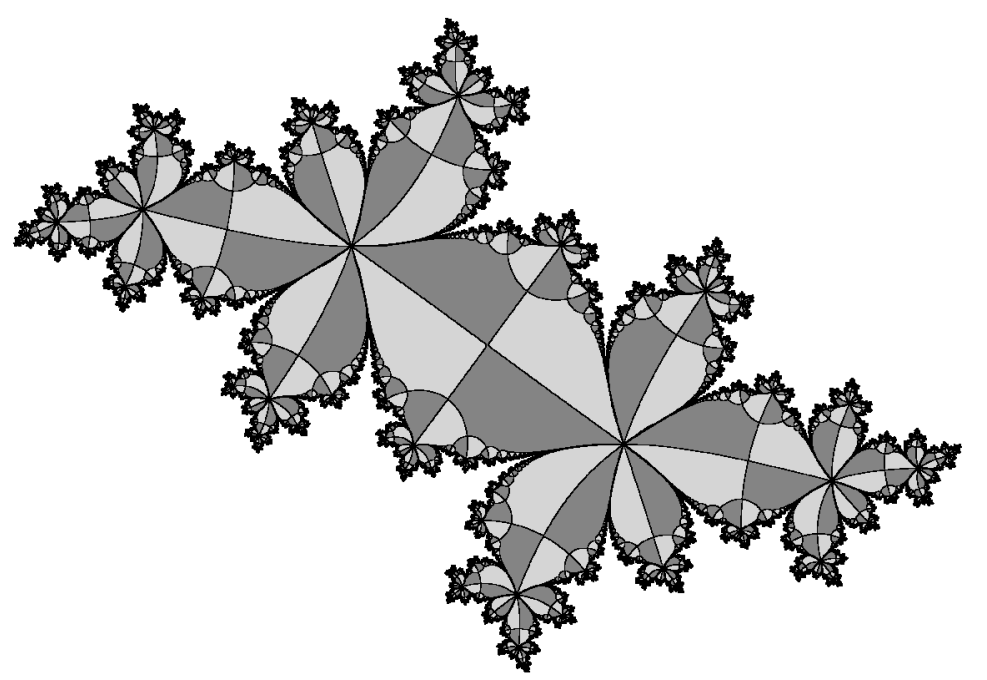

Figure 7. The parabolic chessboard of the Julia set in initial coordinates, for $p / q=2 / 5$ and $\theta=$ the golden mean. 


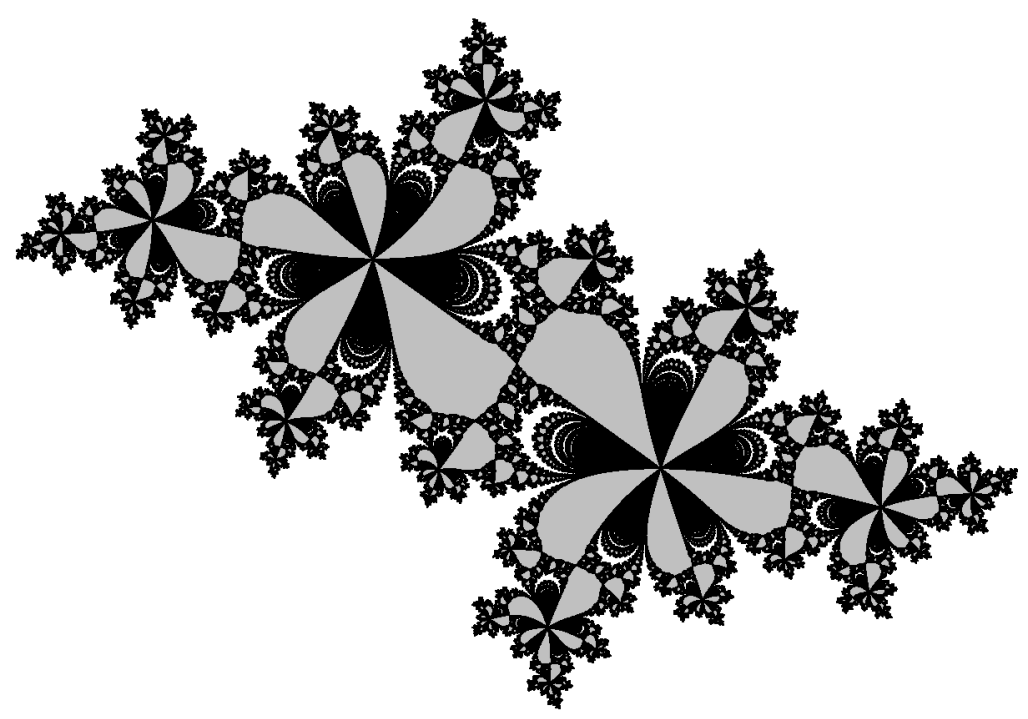

Figure 8 . The Julia-Lavaurs set for $p / q=2 / 5$ and $\theta=$ the golden mean.

\section{UNCOUNTABLY MANY INEQUIVALENT ENTIRE MAPS}

We will provide here an uncountable set of entire maps that are topologically inequivalent, and satisfy the conditions of section 3.2. They will in fact satisfy the stronger following properties: 0 and 1 are critical values, they are the only ones, there are no asymptotic values, 0 has at least one non-critical preimage $z$ such that the component of the preimage of the unit disk that contains $z$ is bounded.

It is enough to construct inequivalent quasiregular maps $f: \mathbb{C} \rightarrow \mathbb{C}$. Indeed, by letting $\mu$ be the pull-back by $f$ of the null Beltrami form, and $\phi$ a straightening $\left(\phi_{*}(\mu)=0\right)$, we obtain an entire map $g=f \circ \phi^{-1}$, equivalent to $f$.

Let $a_{n} \in\{0,1\}$ be indexed by $n \in \mathbb{N}^{*}$. For reasons that will be explained below, we require that $a_{n}=1$ infinitely many times. For $n \in \mathbb{Z}$, let $S_{n}$ be the strip defined by " $\Re(z) \in[n, n+1]$ ". We will define below two continuous maps $A$ and $B$ from $S_{0}$ to $\mathbb{C}$, quasiregular in the interior, and such that $\forall y \in \mathbb{R}, A(i y)=A(1+i y)=$ $B(i y)=B(1+i y)$. Then $f(z)$ will be defined by $f(z)=A(z-n)$ or $f(z)=B(z-n)$ for $z \in S_{n}$, according to the following rule:

- if $n<0$, then we take $A$.

- if $n=0$, then we take $B$.

- if $n>0$, then we take $A$ if $a_{n}=0$, and $B$ if $a_{n}=1$.

Let us now define these maps $A$ and $B$. Let

$$
B(z)=1-(\cos (\pi z))^{4} .
$$

Note that $B$ is holomorphic. It is illustrated by the following picture. 

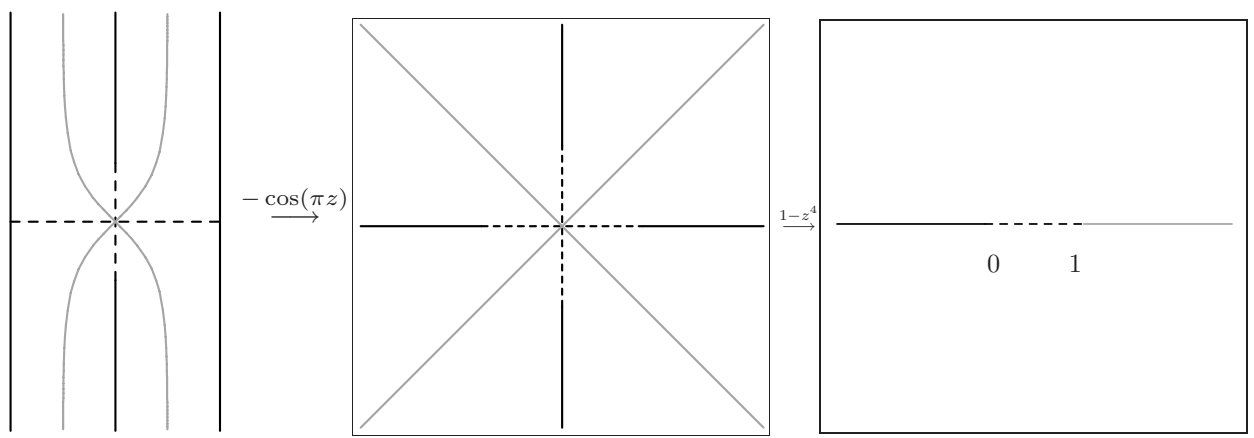

Let

where

$$
A(z)=\frac{1-\cos (2 \pi h(z))}{2}
$$

$$
h(x+i y)=x+i l(y)
$$

and $l$ is an increasing homeomorphism of $\mathbb{R}$ such that $A$ and $B$ coincide on $i \mathbb{R}$.

An explicit computation yields

$$
1-(\cosh (\pi y))^{4}=\frac{1-\cosh (2 \pi l(y))}{2}
$$

from which one deduces that $l$ is a diffeomorphism, with derivative satisfying $l^{\prime}(z) \in$ $[\sqrt{2}, 2[$. Thus $A$ is $K$-quasiconformal with $K=2$.

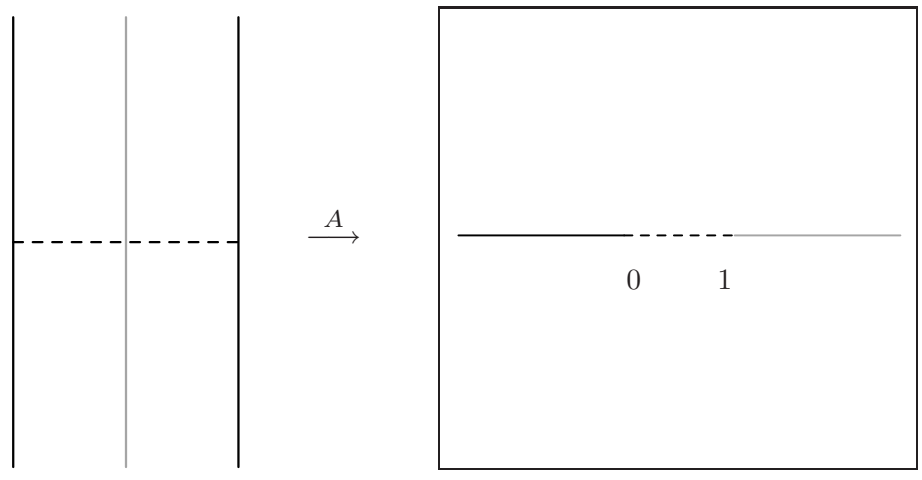

Now let us prove that two different sequences $a_{n}$ yield two inequivalent maps. Let us consider any simple path $\gamma$ from 0 to 1 , in $\mathbb{C} \backslash\{0,1\}$. Any two such paths are isotopic. As a consequence, the preimage of the support of $\gamma$ yields a graph embedded in the complex plane, with vertices labeled 0 or 1 , that is independent of $\gamma$ up to isotopy. We will call this graph the skeleton:

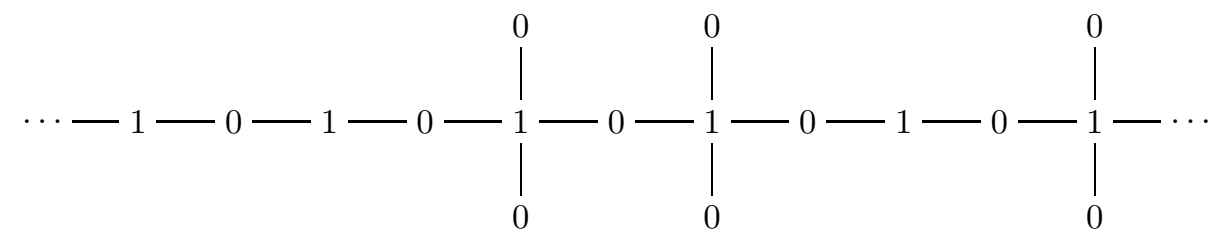

This graph has a spine, a bi-infinite sequence of vertices alternately labeled 0 and 1 , each linked to the other. All the 1 s are on the spine, let us call them the vertebrae. 
Some vertebrae have ribs, i.e. edges that end with lone vertices labeled 0 ; these are the vertebrae associated to the map $B$. The spine has no ribs before the one associated to $n=0$, it has one there, and infinitely many ribs after, since we required that $a_{n}=1$ for infinitely many $n>0$. Equivalent maps must have homeomorphic skeletons, with the homeomorphism respecting labeled points (in fact, even better than that, the embedding in the plane must be isotopic). But since it is possible to topologically identify which $n \in \mathbb{Z}$ is associated to each vertebra, this implies equivalent maps have the same sequence $\left(a_{n}\right)$.

The conclusion follows, because there are uncountably many sequences $a \in$ $\{0,1\}^{\mathbb{N}^{*}}$ with infinitely many $1 \mathrm{~s}$.

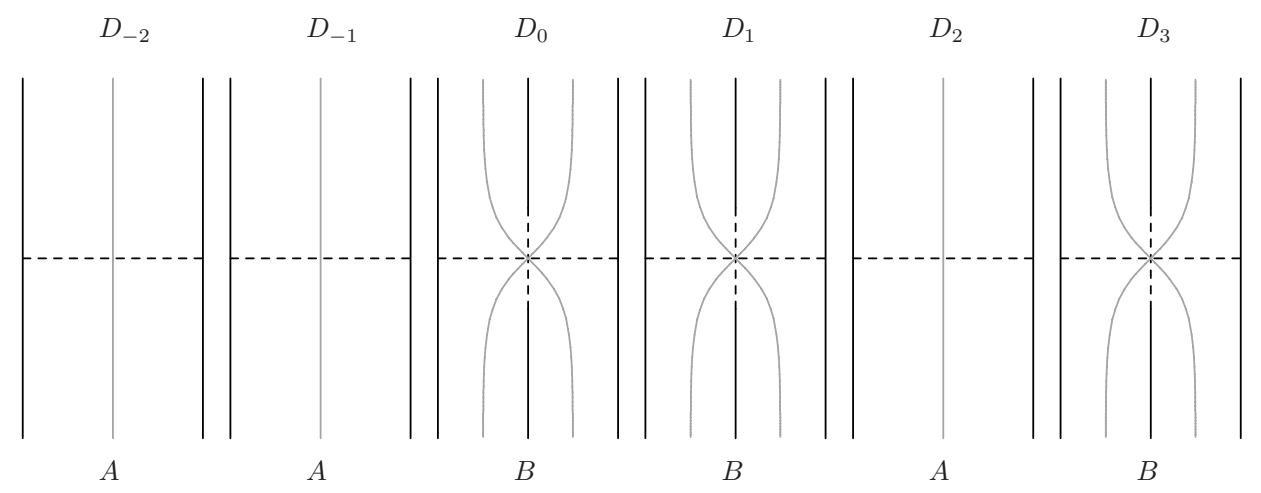

Figure 9. Example of the sequence for the map $f$.

Remark. In fact, one can construct the entire maps $g$ by gluing pieces of ramified coverings, but to prove that the Riemann surface thus constructed is isomorphic to $\mathbb{C}$ and not to $\mathbb{D}$, it is enough to prove it is quasiconformally equivalent to $\mathbb{C}$, which amounts to directly defining a quasiregular map $f$.

Remark. More generally, simply connected ramified covers over $\mathbb{C}$, ramified only over 0 and 1, are completely classified by their skeleton (up to a positive homeomorphism of the plane in which the skeleton is drawn), which can be any finite or infinite tree in the plane (connected graph with no cycles), with no accumulation, and with at least one edge. Rigorously, one needs to label the vertices alternately by 0 and 1 , but note that any unlabeled skeleton has exactly two labelings. When is the Riemann surface thus constructed isomorphic to $\mathbb{C}$ instead of $\mathbb{D}$ ? Is it possible to decide it from the skeleton? The answer of this difficult problem has been given by Doy.

\section{Getting more COntrol For Lavaurs maps}

The notion of $c$-quasisymmetric function which is used here is not invariant by Möbius transformations of $\mathbb{S}^{1}$ for a given value of $c>1$ : By definition, an orientation preserving homeomorphism $f: \mathbb{T} \rightarrow \mathbb{T}$ is $c$-quasisymmetric if and only if, calling $\mathbf{f}$ a lift of $f$ to a homeomorphism of $\mathbb{R}$, we have

$$
\forall x \in \mathbb{R}, \forall h \in] 0,1\left[, c^{-1} \leq \frac{\mathbf{f}(x+h)-\mathbf{f}(x)}{\mathbf{f}(x)-\mathbf{f}(x-h)} \leq c .\right.
$$


Proposition 8.1 (uniformity in the Herman, Świątek theorem). In theorem 4.1, the constant $c$ only depends on the following data:

$$
M, W, l, A \text { and } M^{\prime} \text {. }
$$

See [P2], theorems 1.2 and 1.3.

Proposition 8.2 (uniformity in the Ahlfors, Beurling theorem). There exists a function $K(k):] 1,+\infty[\rightarrow] 1,+\infty[$, such that $K(k) \longrightarrow 1$ when $k \longrightarrow 1$, and such that every $k$-quasisymmetric homeomorphism $f$ of the unit circle extends to a homeomorphism $F$ from $\overline{\mathbb{D}}$ to $\overline{\mathbb{D}}, K$-quasiconformal in $\mathbb{D}$, and that moreover fixes 0 .

Proof. The statement is in [ABe, except for the "fixing 0" claim. For any given $k$ and $K$, the class of $K$-quasiconformal homeomorphisms of $\mathbb{D}$ that extends to a $k$-quasisymmetric homeomorphism of the unit circle, is compact with respect to uniform convergence on $\mathbb{D}$. In particular, the image of 0 by such a homeomorphism belongs to some compact $C$ of $\mathbb{D}$, which depends only on $k$ and $K$. It is then possible to map it to 0 by post-composing with a $C^{\infty}$-diffeomorphism of $\mathbb{D}$ that is the identity on a neighborhood $V$ of $\partial \mathbb{D}$, and whose dilatation is bounded by $K^{\prime}$, where $V$ and $K^{\prime}$ only depend on $C$.

We now need to introduce the following Blaschke product:

$$
\mathcal{P}(z)=\frac{3 z^{2}+1}{z^{2}+3} .
$$

It has degree 2 , leaves $\mathbb{D}, \mathbb{S}^{1}$ and $\mathbb{S}^{2} \backslash \overline{\mathbb{D}}$ invariant, and restricts to a self-covering of $\mathbb{S}^{1}$ of degree 2 . It has a unique fixed point $z=1$, which is parabolic with multiplier 1 with 2 attracting axes, $[1,+\infty[$ and $]-\infty, 1]$. The basin of attraction of the left axis is $\mathbb{D}$ and the basin of the right axis is $\mathbb{S}^{2} \backslash \overline{\mathbb{D}}$. Thus, the Julia set is $\mathbb{S}^{1}$.

It has a horn map that we will denote by $\mathfrak{h}$. It is defined on $\mathbb{C} \backslash \mathbb{R}$ and maps to all of $\mathbb{C}$. The following picture illustrates the parabolic chessboard of this map on the half-plane $\mathbb{H}$. It is followed by the chessboard of a connected component of the domain of definition of the horn map $h$ of the polynomial $P_{p / q}$ (for $p / q=2 / 5$ ). They are linked by the following proposition.
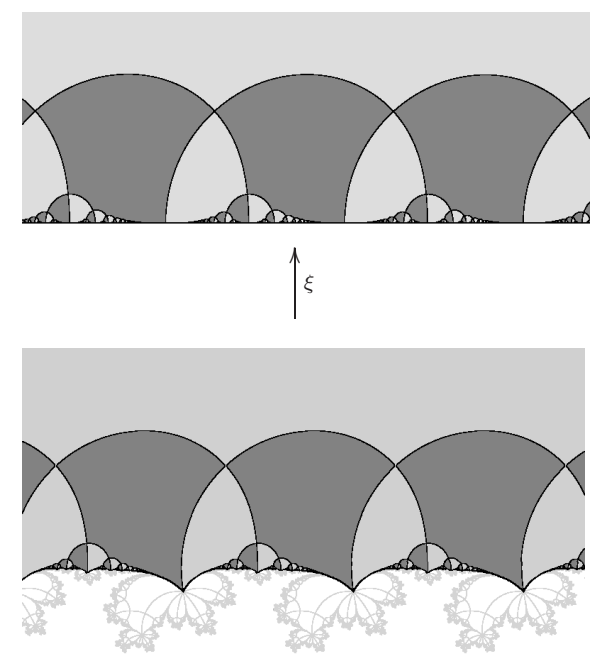
Proposition 8.3 (universality of horn maps as coverings). For all $p / q$, there exists a $\sigma_{0}$ depending on the Fatou coordinates, and an isomorphism $\xi$ from the component $U$ of $\psi_{+}^{-1} \stackrel{\circ}{K}(P)$ that contains an upper half-plane to the upper half-plane $\mathbb{H}$, that commutes with $T_{1}$ and such that

$$
h_{\sigma_{0}}=\mathfrak{h} \circ \xi
$$

holds on $U$.

A proof of this claim can be found in [C]. Basically, it is a transposition to the horn maps of the following universality (see $[\mathrm{DHu}]$ ): For any quadratic polynomial $Q$ having a parabolic point, for any periodic component $C$ of $\stackrel{\circ}{K}(Q)$, let $n$ be the minimal period. Then there is an analytic isomorphism from $C$ to $\mathbb{D}$ conjugating $Q^{n}$ to $\mathcal{P}$.

In particular, proposition 8.3 implies that, modulo $\mathbb{Z}$, the connected component of the domain of definition of the pre-model map $\beta$ that contains the real axis (this is an $\mathbb{R}$-symmetric annulus $A_{p / q}$ ) has modulus equal to $2 m$, where $m$ is the modulus between the upper chessboard box and the boundary of the domain of definition of $h$. This modulus is the same as the corresponding modulus for $\mathfrak{h}$, thus the modulus of $A_{p / q}$ does not depend on $p / q$; it is universal. Let us call $\mathfrak{b}$ the pre-model map constructed from the Blaschke product's horn map $\mathfrak{h}$, let $A$ be its central annulus of definition $(\bmod \mathbb{Z})$, and let $\mathfrak{p}$ be to $\mathfrak{h}$ what $\phi$ is to $h$. Then, the map $\mathfrak{p} \circ \xi \circ \phi^{-1}$ has a Schwarz reflection extension across $\mathbb{R}$ that we will call $\zeta$. It is an isomorphism from the annulus $A_{p / q}$ to $A$. We have

$$
\left.\beta\right|_{A_{p / q}}=\mathfrak{b} \circ \zeta
$$

From this, one deduces that, for a fixed bound $M$ on the entries of the continued fraction expansion of $\theta$, the conditions of proposition 8.1 are uniformly satisfied, i.e. with constants that do not depend on $p / q$. We have thus proved the following theorem.

Theorem 8.1 (universal bound on dilatation ratio). For all $M>0$, there exists $K>0$ such that for all $p / q$ irreducible and all irrational rotation number $\theta$ of type bounded by $M$, the straightenings $S, R$ and s have dilatation ratio bounded by $K$.

Here is an example of a result which follows:

Corollary 8.1. The modulus of the annulus in the cylinder $\mathbb{C} / \mathbb{Z}$ separating $\Delta$ from the boundary of the domain of definition of $h$, is bounded below (and above), independently of $p / q$, by a constant depending only on $M$.

Proof. This annulus is the image by $S$ of the lower half of the annulus $A_{p / q}$, and this half has universal modulus.

\section{OBJECTS OF the PARABOLIC IMPLOSION}

Here, we give the definition of the objects involved in parabolic implosion. 


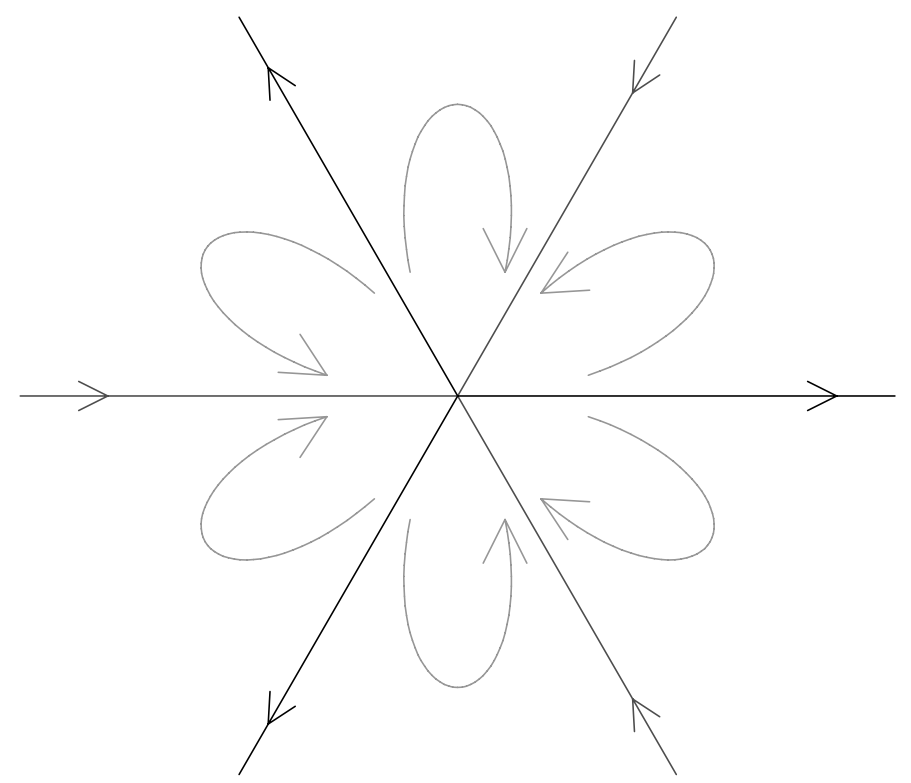

The repelling axes of the parabolic point $z=0$ are labeled by the index $j$, which can take $q$ values. The attracting ones are labeled by the index $i$, which can also take $q$ values. These $2 q$ axes are half-lines starting from 0 , alternatively attracting and repelling, and they all make equal angles. The extended repelling Fatou parameterization associated to the $j$ th axis is denoted by

$$
\psi_{+, j}: \mathbb{C}_{j} \rightarrow \mathbb{C}
$$

where $\mathbb{C}_{j}$ is just a labeled copy of $\mathbb{C}$. It is a holomorphic map, semi-conjugating $T_{1}$ to $P^{q}$, i.e. the following diagram commutes:

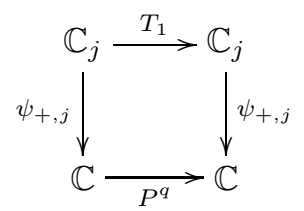

The extended attracting Fatou coordinate will be denoted by

$$
\phi_{-, i}: \stackrel{\circ}{K}_{i} \rightarrow \mathbb{C}_{i}
$$

where $\stackrel{\circ}{K}_{i}$ stands for the set of points in $\mathbb{C}$ attracted by the $i$ th axis under the dynamics of $P^{q}$. The union of the $\stackrel{\circ}{K}_{i}$ is disjoint, and is equal to $\stackrel{\circ}{K}$. We have the following commutative diagram:

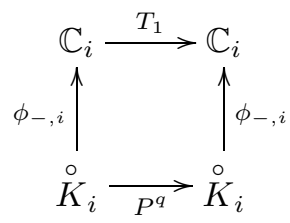

Moreover, $P$ acts on the set of repelling (resp. attracting) axes, like a rotation of $p / q$ turns. Let us denote by $i^{P}$ the $i$ th axis maps to, and $j^{P}$ the $j$ th axis maps to. 
We have the following commutative diagrams:
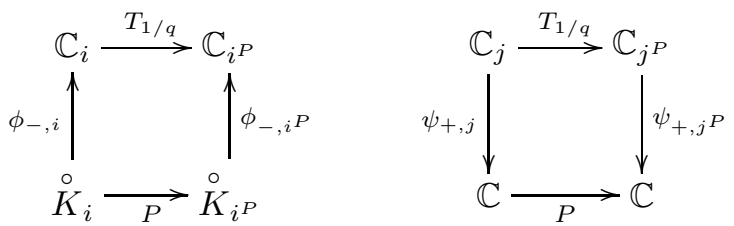

With these properties, the Fatou coordinates and parameterizations are all unique up to a composition with two translations: More precisely, the $\psi_{+, j}$ may be all pre-composed with the same translation, and the $\phi_{-, i}$ may be all post-composed with another common translation. (Thus there are two $\mathbb{C}$-degrees of freedom in the choices.)

We introduce the maps

$$
\phi_{\dot{\div}, i}: \stackrel{\circ}{K} \rightarrow \mathbb{C}_{i}
$$

by the formula

$$
\forall i, \forall i^{\prime}, \forall z \in \stackrel{\circ}{K}_{i^{\prime}}, \phi_{\div, i}(z)=\phi_{-, i} \circ P^{k}(z)=T_{\frac{k}{q}} \circ \phi_{-, i^{\prime}}
$$

where $k \geq 0$ is the smallest number of time $P$ must be iterated to map the $i^{\prime}$ th axis to the $i$ th axis: $k$ depends on $i$ and $i^{\prime}$. (Note that the definition is slightly different than the one in $\left[\mathrm{C}\right.$, since here our map $\phi_{\div, i}$ is indexed by $i$ instead of $j$, and we do not include the translation $T_{\sigma}$.)

Let us mention the following relation:

$$
\forall i, \forall z \in \stackrel{\circ}{K}, \phi_{\div, i} \circ P(z)=\phi_{\div, i}(z)+m_{i, z}
$$

where, if we call $i^{\prime}$ the index such that $z \in \stackrel{\circ}{K}_{i^{\prime}}$, we define $m_{i, z}=1$ if $i^{\prime}=i$ and $m_{i, z}=0$ else.

In parabolic implosion, one must choose $\nu \in\{-1,1\}$, which stands for the direction of the implosion, positive or negative. If $\nu=1$, we define $j(i)$ to be the index of the repelling axis right after the attracting axis labeled $i$ in cyclic order. If $\nu=-1$, we define $j(i)$ as the index of the repelling axis just before the $i$ th axis. The inverse map will be denoted by $i(j)$. The choice of the direction of the implosion is not to be mistaken with the choice of the end of the cylinder where we put the Siegel disk.

To simplify notations, we choose an index $j=j_{0}$, fix it, and we write $\mathbb{C}_{+}=\mathbb{C}_{j_{0}}$ and $\mathbb{C}_{-}=\mathbb{C}_{i\left(j_{0}\right)}$. The notation $\psi_{+}$will stand for $\psi_{+, j_{0}}$, and $\phi_{\div}$for $\phi_{\div, i\left(j_{0}\right)}$. Let us consider the following non-commutative diagram

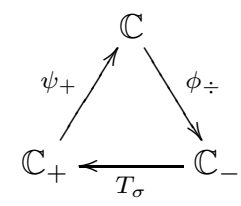

where $\sigma$ is a parameter: $\sigma \in \mathbb{C}$.

The modified Lavaurs maps are denoted by

$$
g_{\sigma}: \stackrel{\circ}{K} \rightarrow \mathbb{C} .
$$


They are defined by following the triangular diagram from the vertex labeled $\mathbb{C}$ to itself. In other words, they are defined by the formula

$$
g_{\sigma}=\psi_{+} \circ T_{\sigma} \circ \phi_{\doteqdot} .
$$

The extended horn maps are defined by following the diagram, this time starting from $\mathbb{C}_{+}$. In other words,

$$
h_{\sigma}=T_{\sigma} \circ \phi_{\div} \circ \psi_{+} .
$$

Thus they are defined on the subset $\phi_{+}^{-1}(\stackrel{\circ}{K})$ of $\mathbb{C}_{+}$:

$$
\left.h_{\sigma}: \phi_{+}^{-1} \stackrel{\circ}{K}\right) \rightarrow \mathbb{C}_{+}
$$

The maps $g$ and $h$, for the same value of $\sigma$, are semi-conjugated to each other, as can be derived from either the triangle or the formulas: $g_{\sigma} \circ \psi_{+}=\psi_{+} \circ h_{\sigma}$ and $h_{\sigma} \circ\left(T_{\sigma} \circ \phi_{\doteqdot}\right)=\left(T_{\sigma} \circ \phi_{\doteqdot}\right) \circ g_{\sigma}$.

Remark. The definition of the non-modified Lavaurs map is just slightly more complicated:

$$
\forall i, \forall z \in \stackrel{\circ}{K}_{i}, L_{\sigma}(z)=\psi_{+, j(i)} \circ T_{\sigma} \circ \phi_{-, i}(z) .
$$

This definition does not single out any particular value of $j$. Now we have

$$
\forall i, \forall z \in \stackrel{\circ}{K}_{i}, g_{\sigma}(z)=P^{k} \circ L_{\sigma}(z)
$$

where $k$ is the smallest non-negative iterate of $P$ that maps the $i$ th axis to $i\left(j_{0}\right)$ th axis.

\section{Conclusion}

The model should enable proving local connectivity and zero measure of the Julia-Lavaurs set of quadratic polynomials, and also help study the case of some entire maps.

The construction we propose can be carried out for much more general maps, to give other pre-models. But we are not always guaranteed that the straightening gives the same map we started from. It would be interesting to study the case where there is less rigidity, and/or more singular values.

\section{ACKNOWLEDGMENTS}

This work, except for the entire maps, is part of my Ph.D. thesis. I would like to thank my advisor Adrien Douady. I would like to thank Nuria Fagella and Lukas Geyer for helpful discussions. I would like to thank Dierk Schleicher who helped me to determine for which class of entire maps my construction works. I would also like to thank Marguerite Flexor for the uncountably many times she read different preliminary versions of this work.

\section{REFERENCES}

[ABe] L. V. Ahlfors, A. Beurling, The boundary correspondence under quasiconformal mappings, Acta Mathematica, Vol. 96 (1956), 125-142. MR0086869 (19:258c)

[Br] A.D. Brjuno, Analytical form of differential equations, Trans. Mosc. Math. Soc. 25 (191), 131-288.

[C] A. Chéritat, Recherche d'ensembles de Julia de mesure de Lebesgue positive, Thèse, université Paris-sud, France, 2001.

[D1] A. Douady, Disques de Siegel et anneaux de Herman, Séminaire Bourbaki, Vol. 1986/87, Astérisque No. 152-153 (1987), 4, 151-172 (1988). MR0936853 (89g:30049) 
[D2] A. Douady, Does a Julia Set Depend Continuously on the Polynomial? In: "Complex dynamical Sytems, The Mathematics behind the Mandelbrot and Julia Sets", R.L. Devaney, Proceedings of Symposia in Applied Mathematics, Vol. 49, American Mathematical Society, 1994. edition : 1995. MR.1315535

[DE] A. Douady, C. J. Earle, Conformally natural extension of homeomorphisms of the circle, Acta Mathematica, Vol. 157, (1986), no. 1-2, 23-48. MR0857678 (87j:30041)

[DHu] A. Douady, J.H. Hubbard, Étude dynamique des polynômes complexes, Publications mathématiques d'Orsay, France, 1984-85.

[Doy] P. G. Doyle, Random walk on the Speiser graph of a Riemann surface, Bull. Amer. Math. Soc. (N.S.) 11 (1984), no. 2, 371-377. MR0752802 (86b:58129)

[EL] A. Eremenko, M. Lyubich, Dynamical properties of some classes of entire functions, Annales de l'institut Fourier, tome 42, no. 4 (1992), 989-1020. MR.1196102 (93k:30034)

[Ge] L. Geyer, Siegel discs, Herman rings and the Arnold family, Trans. Amer. Math. Soc. 353 (2001), no. 9, 3661-3683. MR1837254 (2002d:37071)

[Gh] E. Ghys, Transformations holomorphes au voisinage d'une courbe de Jordan, C.R. Acad. Sc. Paris, t. 289 (1984), 383-388.

[He1] M. Herman, Conjuguaison quasi-symétrique des difféomorphismes du cercle et applications aux disques singuliers de Siegel, Manuscrit, 1986.

[He2] M. Herman, Conjugaison quasi symétrique des homéomorphismes du cercle à des rotations, Manuscrit, 1987.

[L] P. Lavaurs, Systèmes dynamiques holomorphes : explosion de points périodiques paraboliques, Ths̀e, Université Paris-Sud, France, 1989.

[mM] C.T. McMullen, Self-similarity of Siegel disks and Hausdorff dimension of Julia sets, Acta Mathematica, Vol. 180, (1998), no. 2, 247-292. MR.1638776 (99f:58172)

[dMvS] W. de Melo, S. van Strien, One-Dimensional Dynamics, Ergebnisse der Mathematik und ihrer Grenzgebiete, 3. Folge · Band 25, Springer-Verlag, 1993. MR1239171 (95a:58035)

[P1] C.L. Petersen, Local Connectivity of some Julia sets containing a circle with an irrational rotation, Acta Mathematica, Vol. 177, 1996, no. 2, 163-224. MR1440932 (98h:58164)

[P2] C.L. Petersen, The Herman-Światek Theorem with applications, London Mathematical Society Lecture Note Series 274: "The Mandelbrot Set, Theme and Variations", Tan Lei, Cambridge University Press, 2000. MR.1765080 (2001a:37004)

[PZ] C.L. Petersen, S. Zakeri, On the Julia Set of a Typical Quadratic Polynomial with a Siegel Disk, Ann. of Math. (2) 159 (2004), no. 1, 1-52. MR2051390 (2005c:37085)

[Rd] B. Rodin, Intrinsic rotations of simply connected regions, Complex Variables. Theory and Application. Vol. 2, pp. 319-326, (1984). MR0743955 (85m:30026)

[Rg] J.T. Rogers, Is the boundary of a Siegel disk a Jordan curve? Bulletin of the American Mathematical Society, Vol. 27, no. 2, pp. 284-287, Oct. 1992. MR.1160003 (93g:30009)

[Si] C. L. Siegel, Iteration of analytic functions, Annals of Mathematics. Second Series. Vol. 43, pp. 607-612, 1942. MR0007044 (4:76c)

[Sh] M. Shishikura, Bifurcation of parabolic fixed points, In: "The Mandelbrot Set, Theme and Variations", L. Tan, London Mathematical Society Lecture Note Series n. 274, Cambridge University Press, 2000. MR 1765097 (2001k:37073)

[Sw] G. Świątek, On critical circle homeomorphisms. Boletim da Sociedade Brasileira de Matemática (Nova Série) 29, no. 2, 1998. 329-351. MR1654840(2000a:37023)

[YaZ] M. Yampolsky, S. Zakeri, Mating Siegel quadratic polynomials, J. Amer. Math. Soc. 14 (2001), no. 1, 25-78. MR.1800348 (2001k:37064)

[Yo] J.C. Yoccoz, Petits diviseurs en dimension 1, Astérisque, Vol. 231, Société Mathématique de France, 1995.

Laboratoire Émile Picard, Université Paul Sabatier, 118 route de Narbonne, 31062 Toulouse Cedex, France 